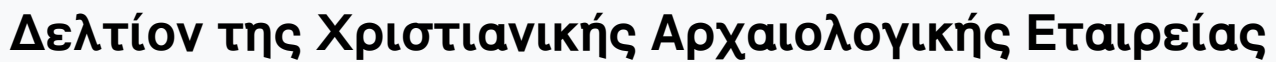

Tó 30 (2009)

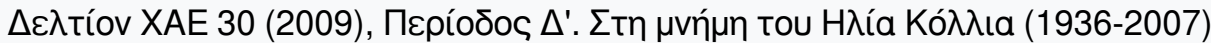

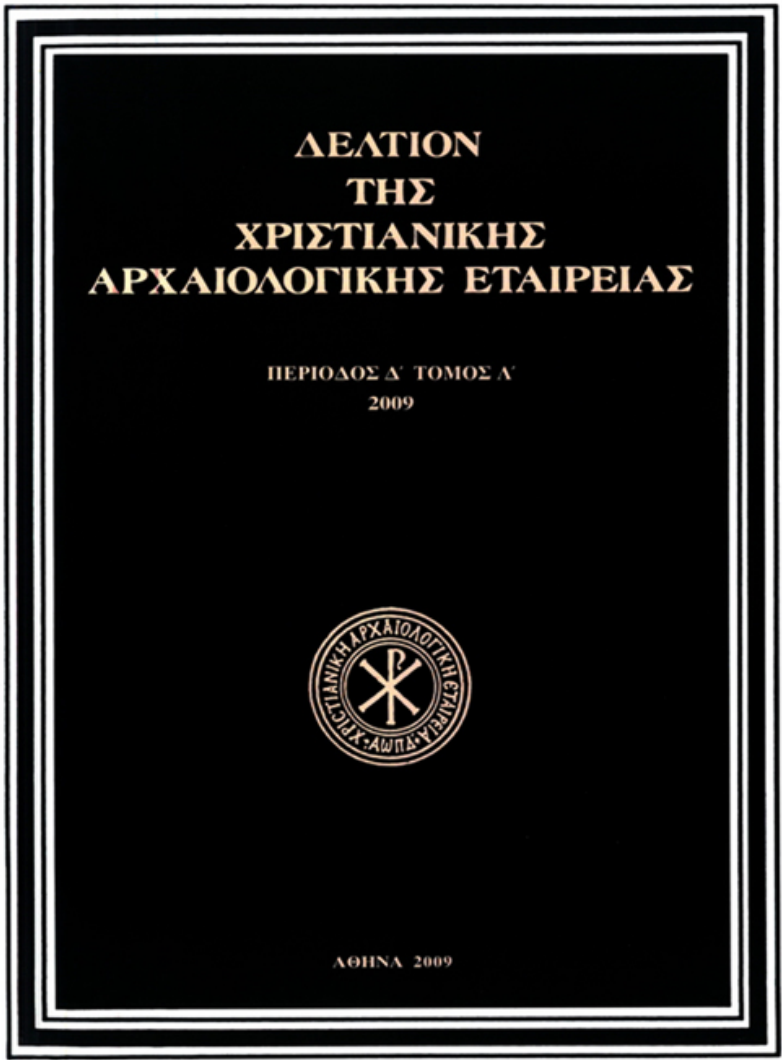

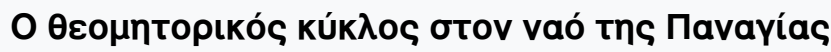

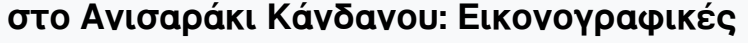

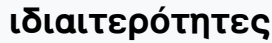

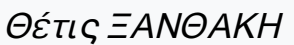

doi: $10.12681 /$ dchae.649

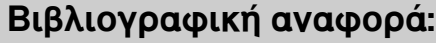

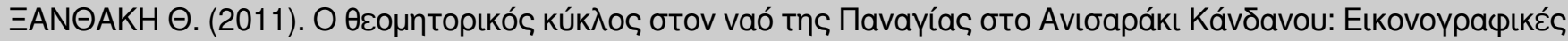

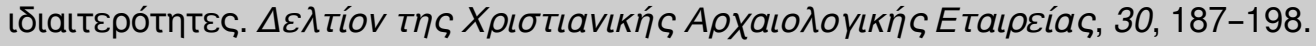

https://doi.org/10.12681/dchae.649 


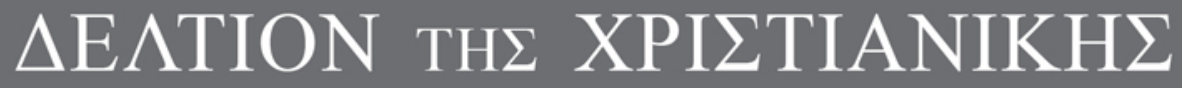 APXAIO $\Lambda$ OГIKH $\Sigma$ ETAIPEIA $\Sigma$}

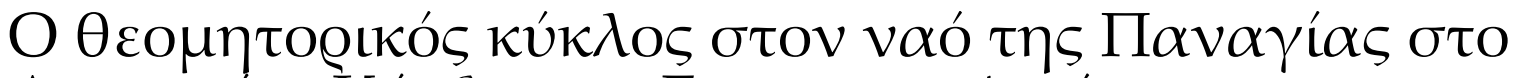

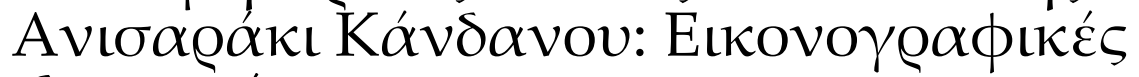

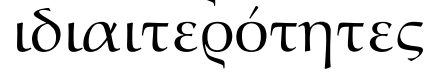

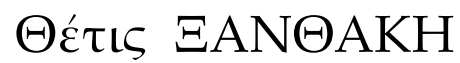

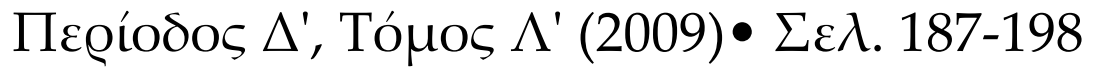
A@HNA 2009 


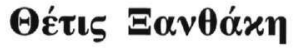

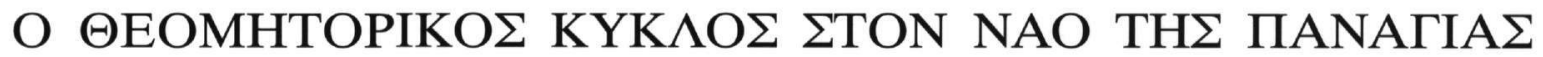 $\Sigma$ TO ANI $\Sigma$ APAKI KAN $\triangle A N O Y$ : EIKONOГPAФIKE $\Sigma$ I $\triangle$ IAITEPOTHTE $\Sigma$}

$\mathrm{O}$

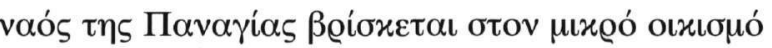

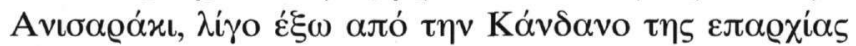

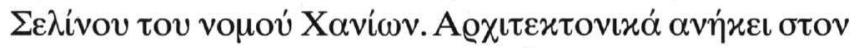

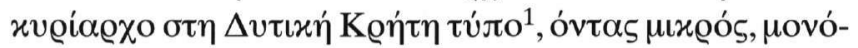

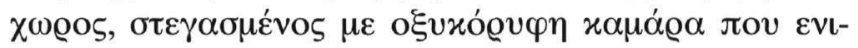

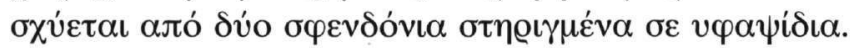

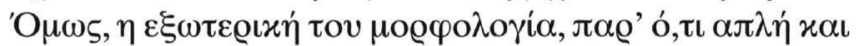

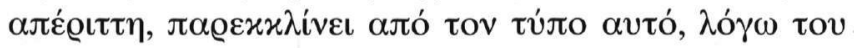

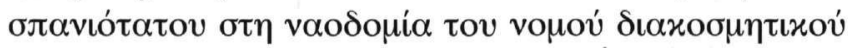

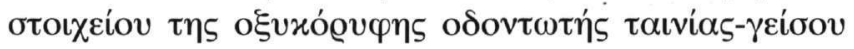

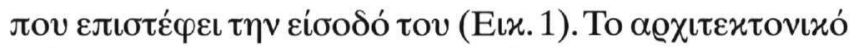

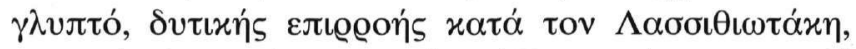

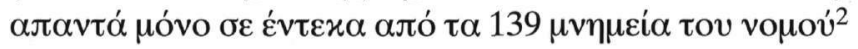

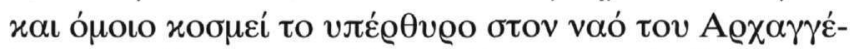

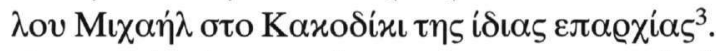

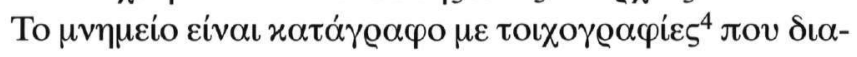

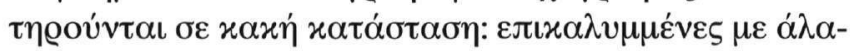

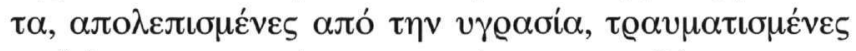

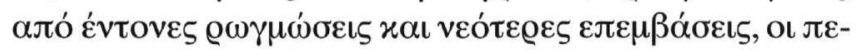

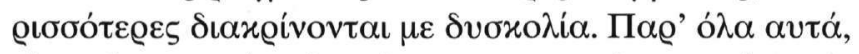

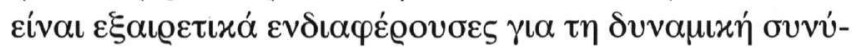

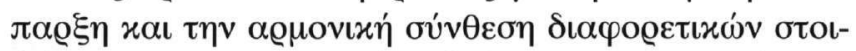

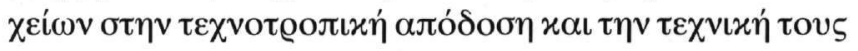

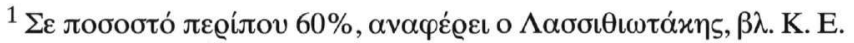

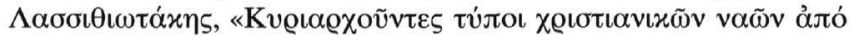

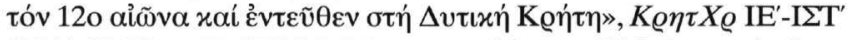

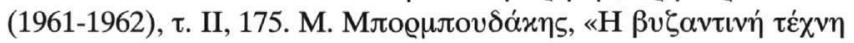

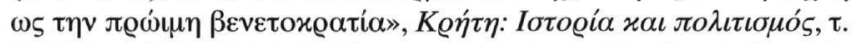

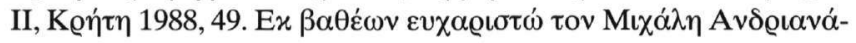

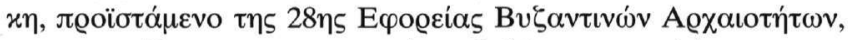

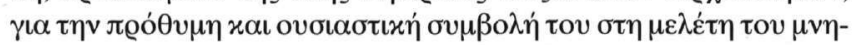

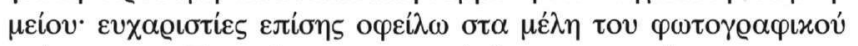

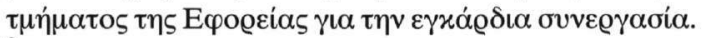

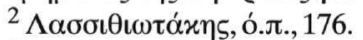

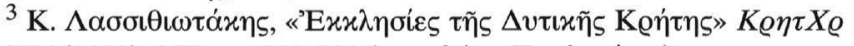

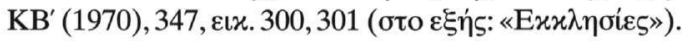

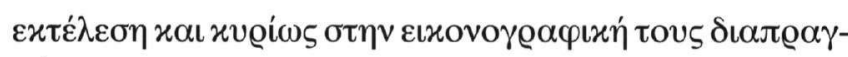

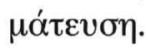

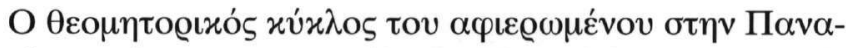

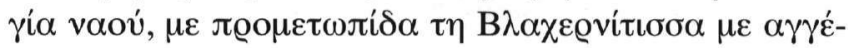

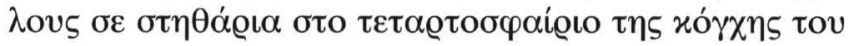

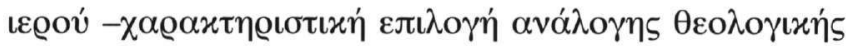

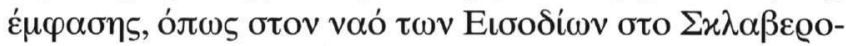

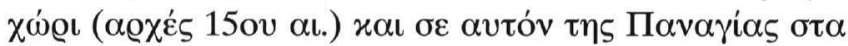

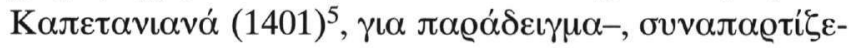

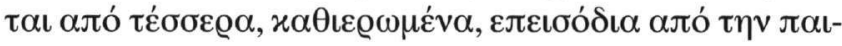

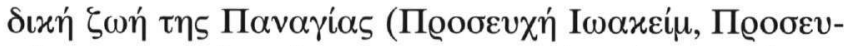

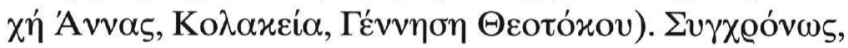

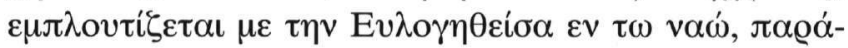

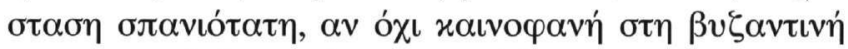

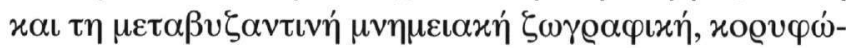

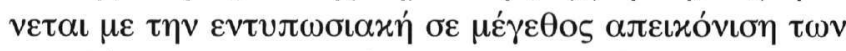

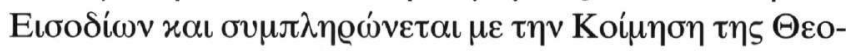

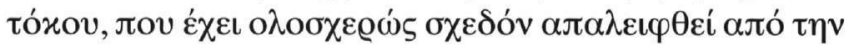

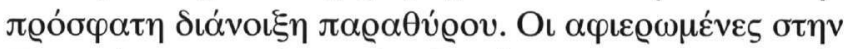

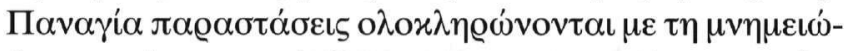

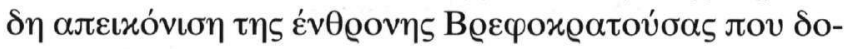

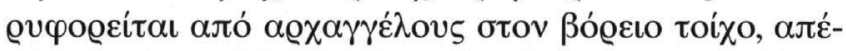

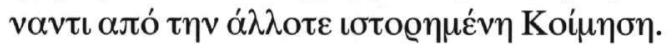

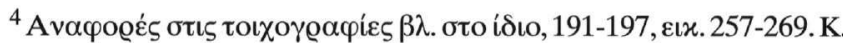
Kalokyris, The Byzantine Wall Paintings of Crete, Néa Yó@xך 1973, 55,

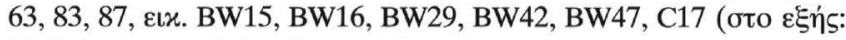
Wall Paintings of Crete). K. Gallas - K. Wessel - M. Borboudakis,

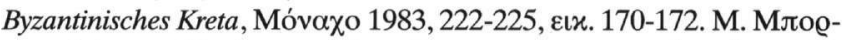

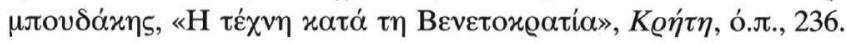

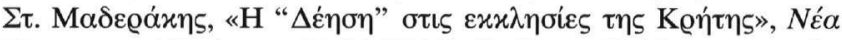

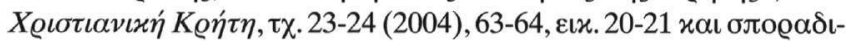

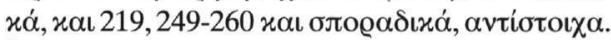

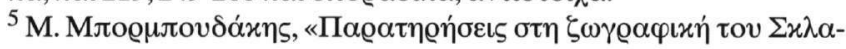

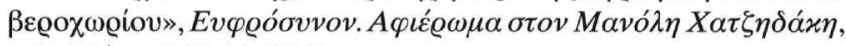

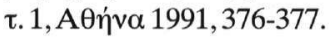




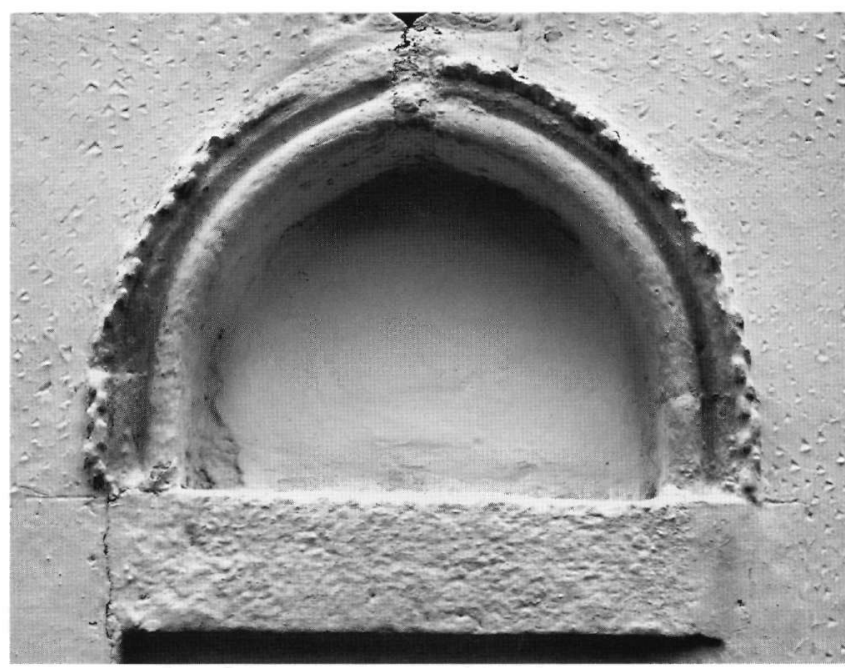

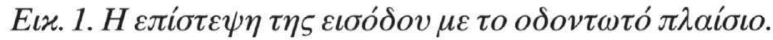

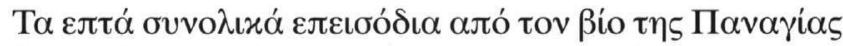

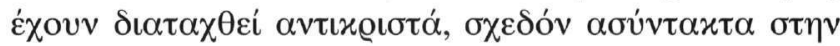

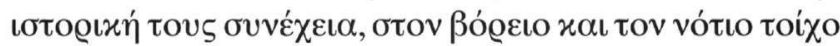

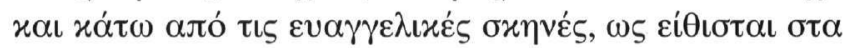

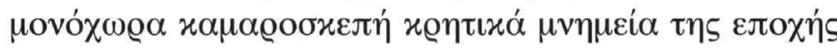

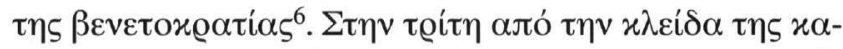

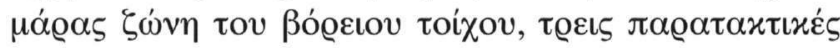

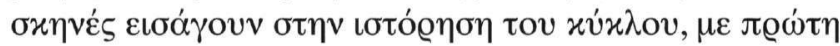

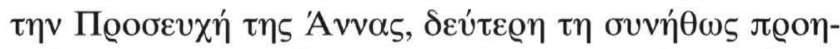

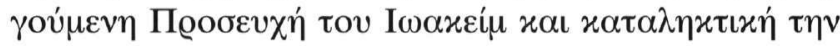

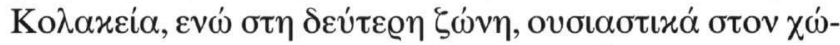

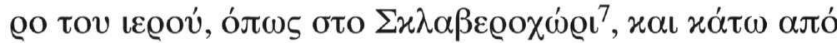

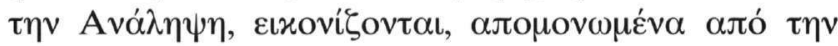

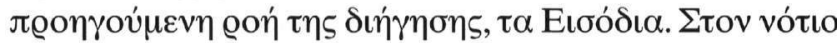

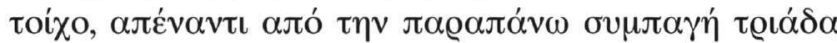

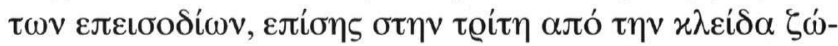

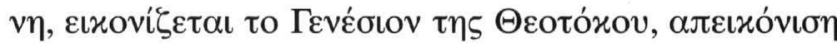

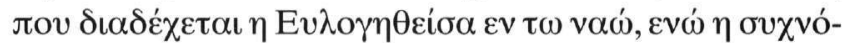

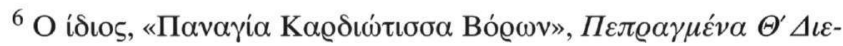

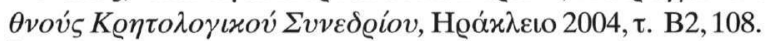

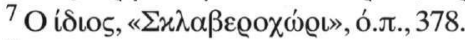

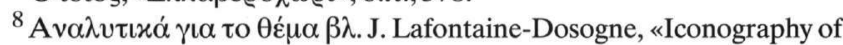
the Cycle of the Virgin», The Kariye Djami, 4. Studies in the Art of the

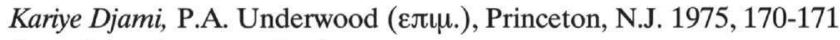
( $\sigma \tau 0 \varepsilon \xi \eta \dot{\eta}$ : «Iconography»).

${ }^{9}$ P.A. Underwood, The Kariye Djami, 2. Plates, The Mosaics, Nع́a Yó@-

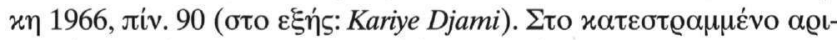

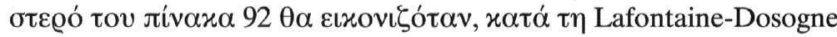

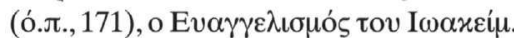

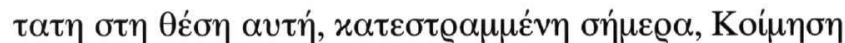

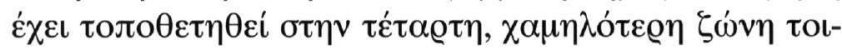

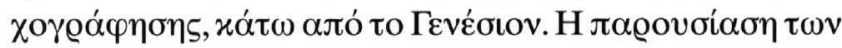

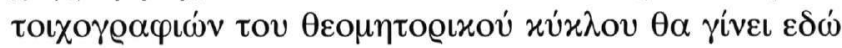

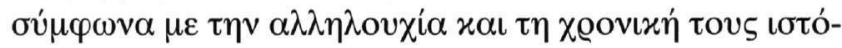

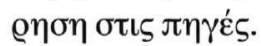

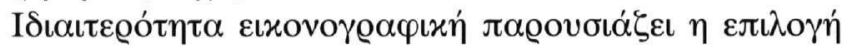

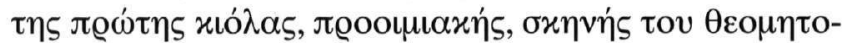

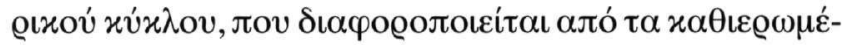

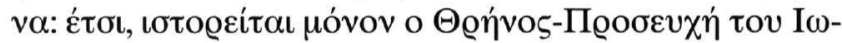

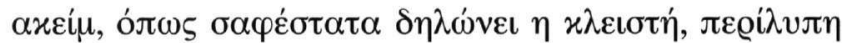

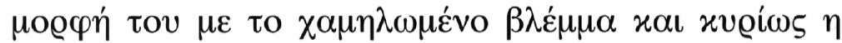

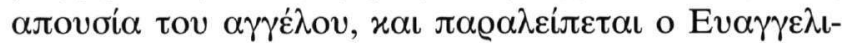

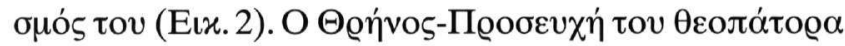

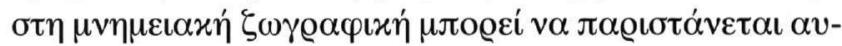

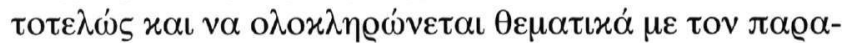

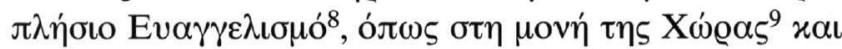

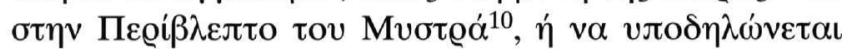

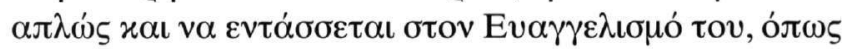

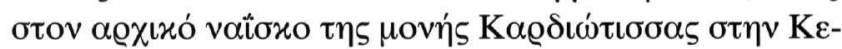

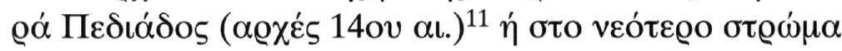

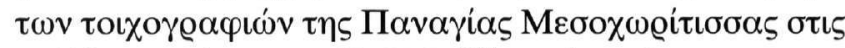

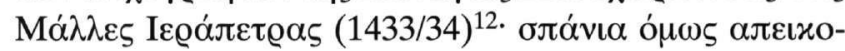

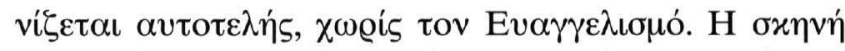

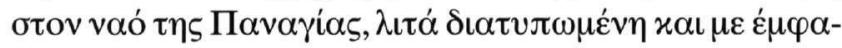

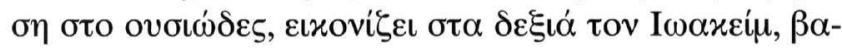

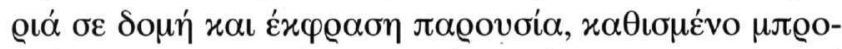

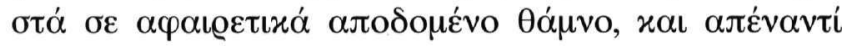

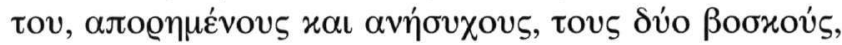

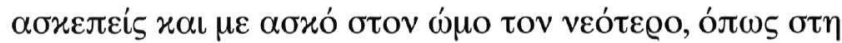

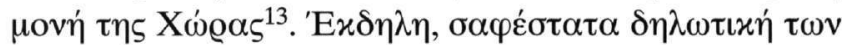

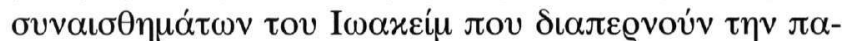

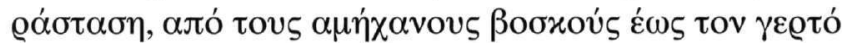

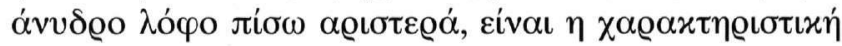

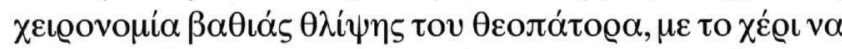

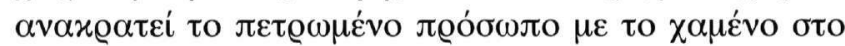

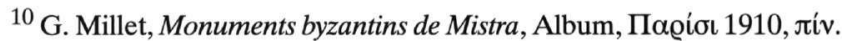
125.3 .

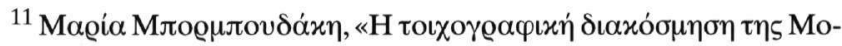

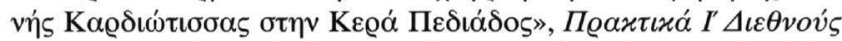

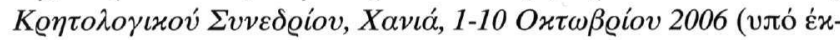

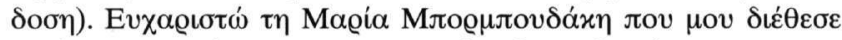

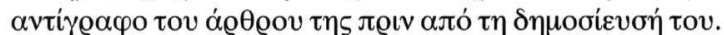

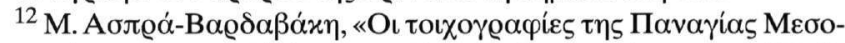

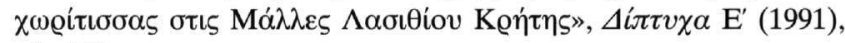
лiv. $19 \beta$.

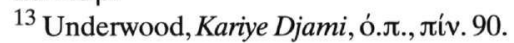




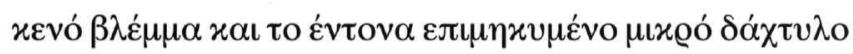

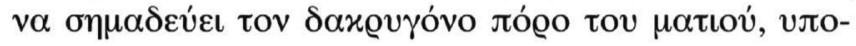

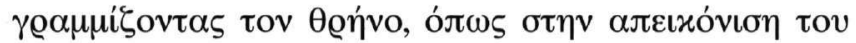

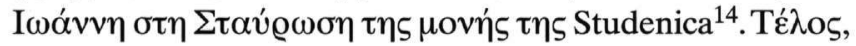

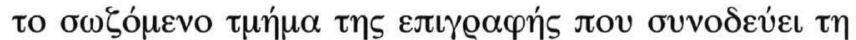

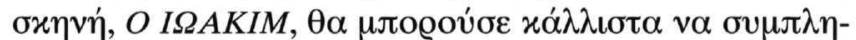

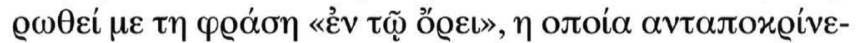

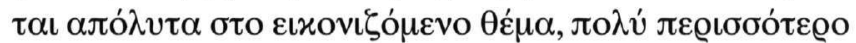

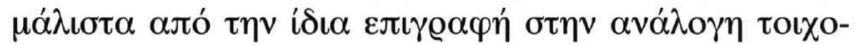

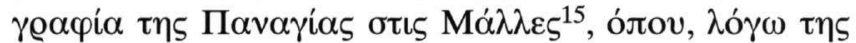

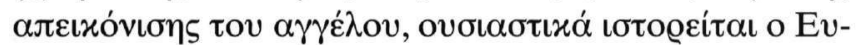

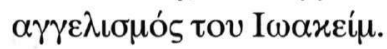

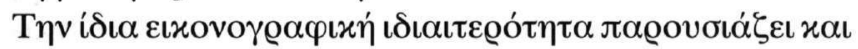

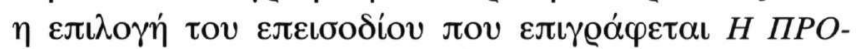

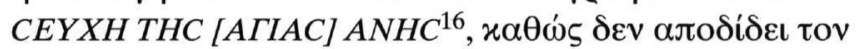

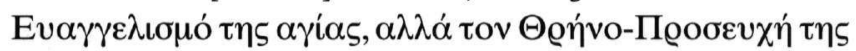

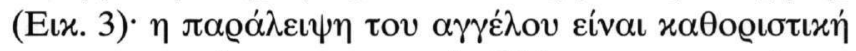

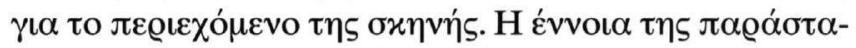

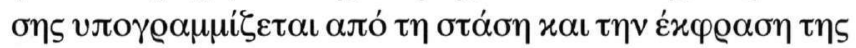

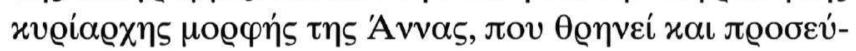

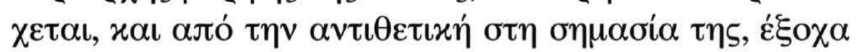

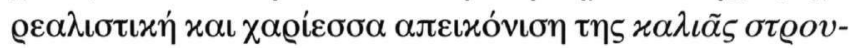

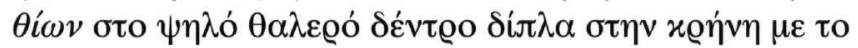

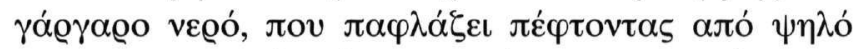

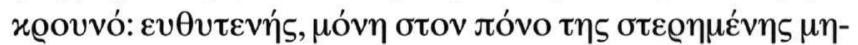

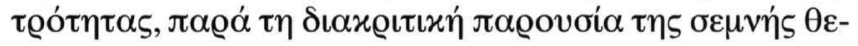

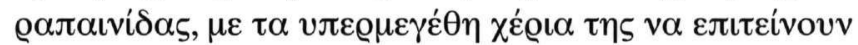

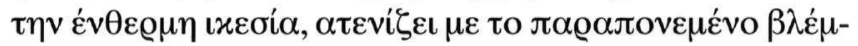

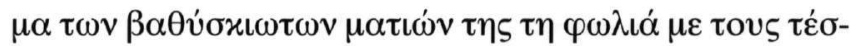

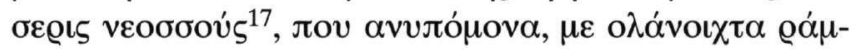

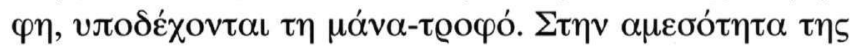

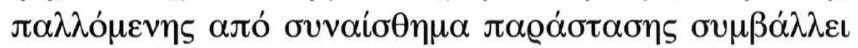

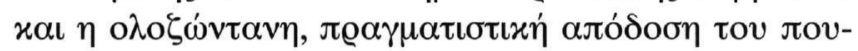

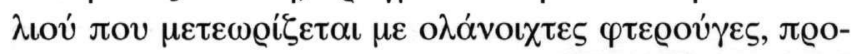

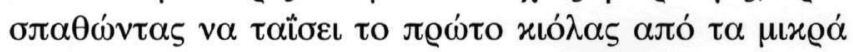

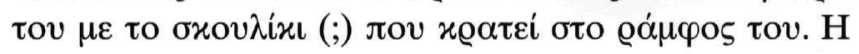

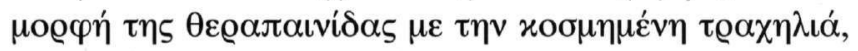

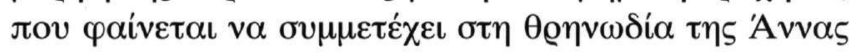

${ }^{14}$ G. Millet - A. Frolow, La peinture du Moyen Âge en Yougoslavie

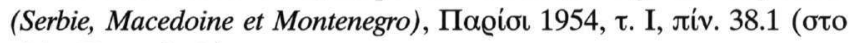
$\varepsilon \xi \dot{\eta} \varsigma:$ Yougoslavie).

${ }^{15}$ I. Spatharakis, Dated Byzantine Wall Paintings of Crete, Leiden 2001,

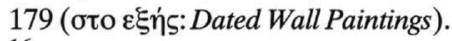

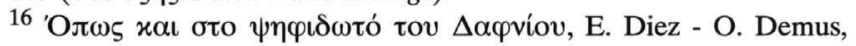
Byzantine Mosaics in Greece: Daphni and Hosios Lucas, Cambridge

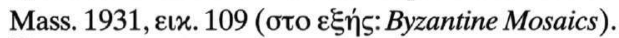

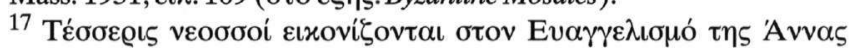

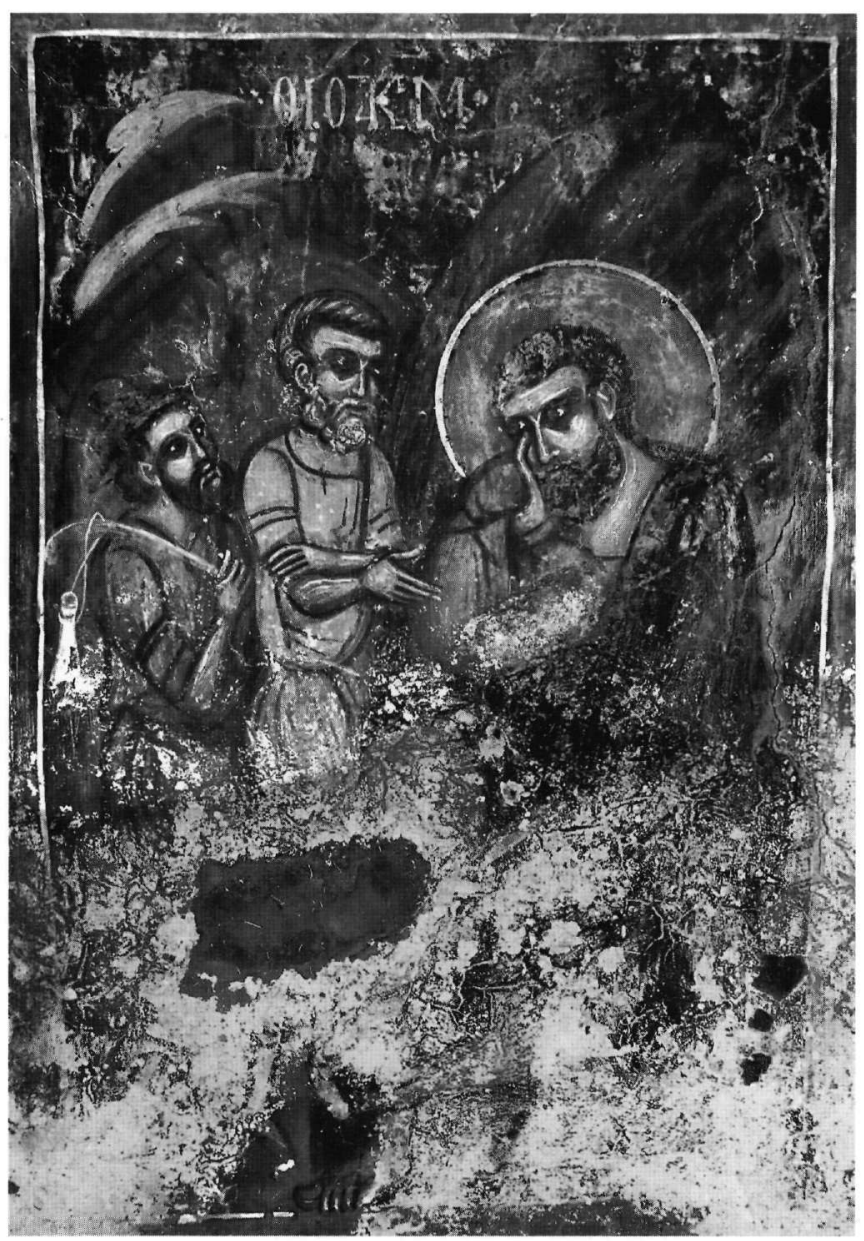

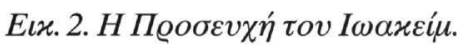

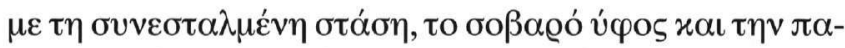

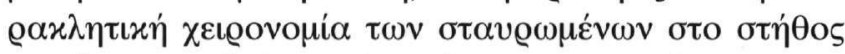

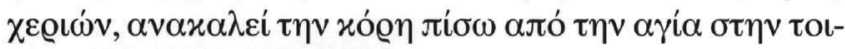

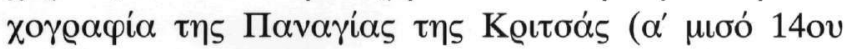

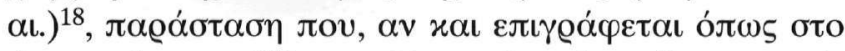

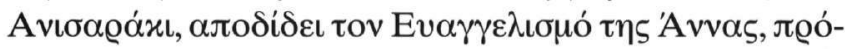

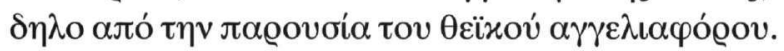

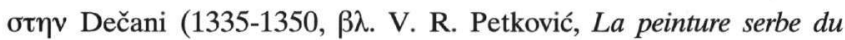

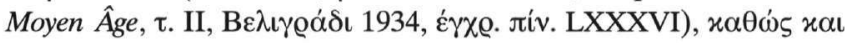

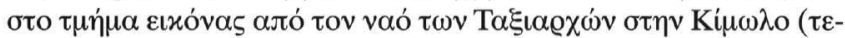

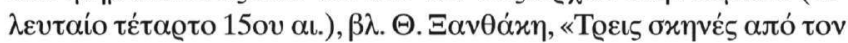

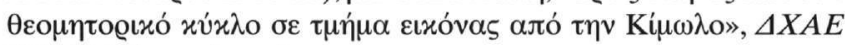

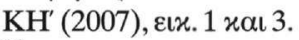

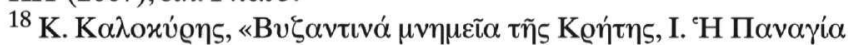

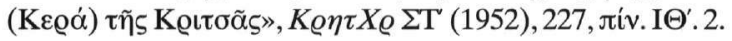




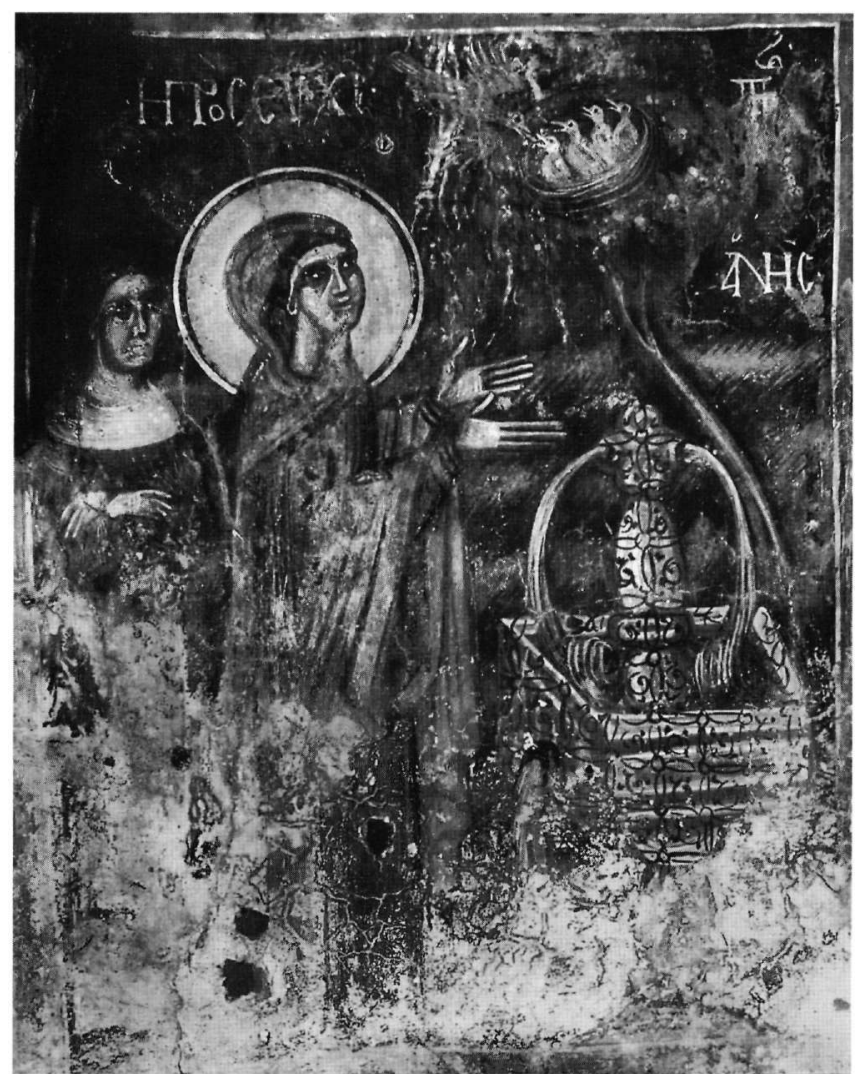

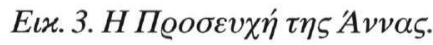

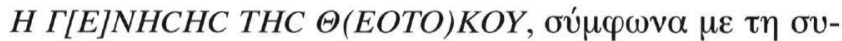

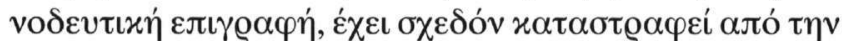

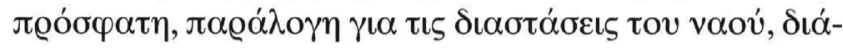

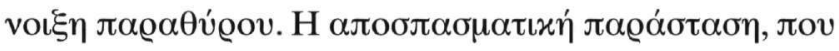

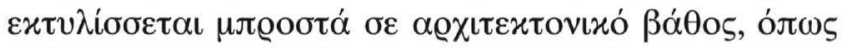

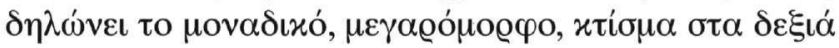

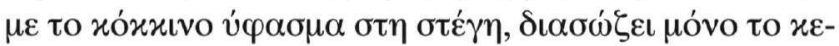

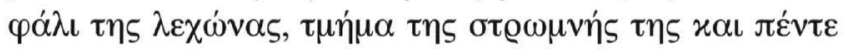

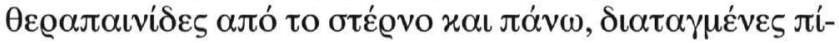

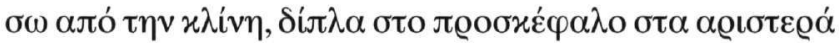

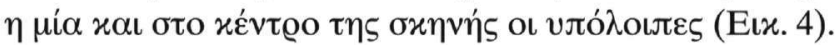

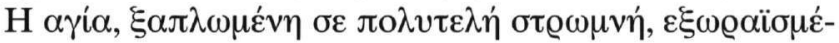

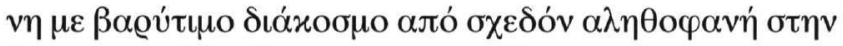

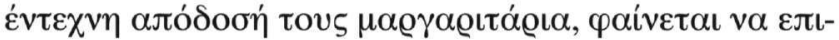

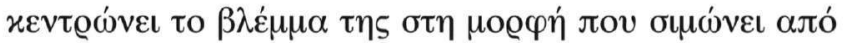

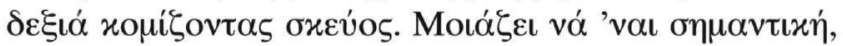

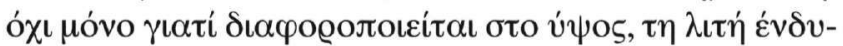

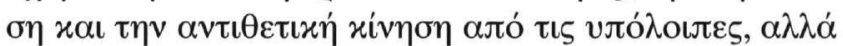

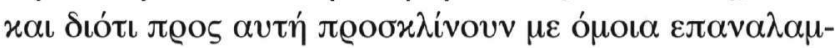

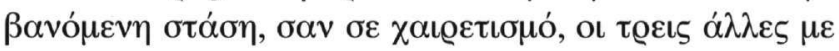

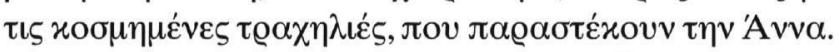

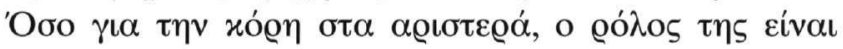

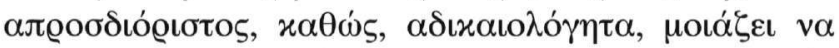

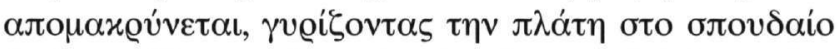

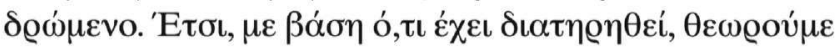

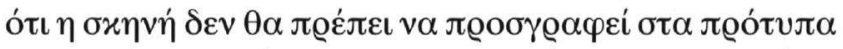

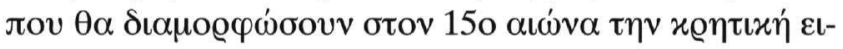

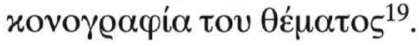

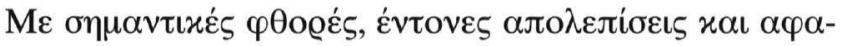

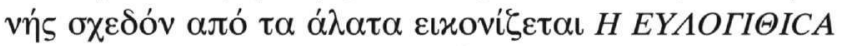

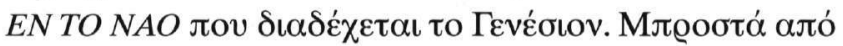

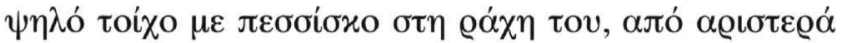

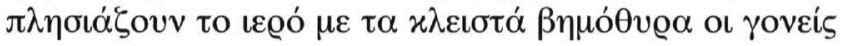

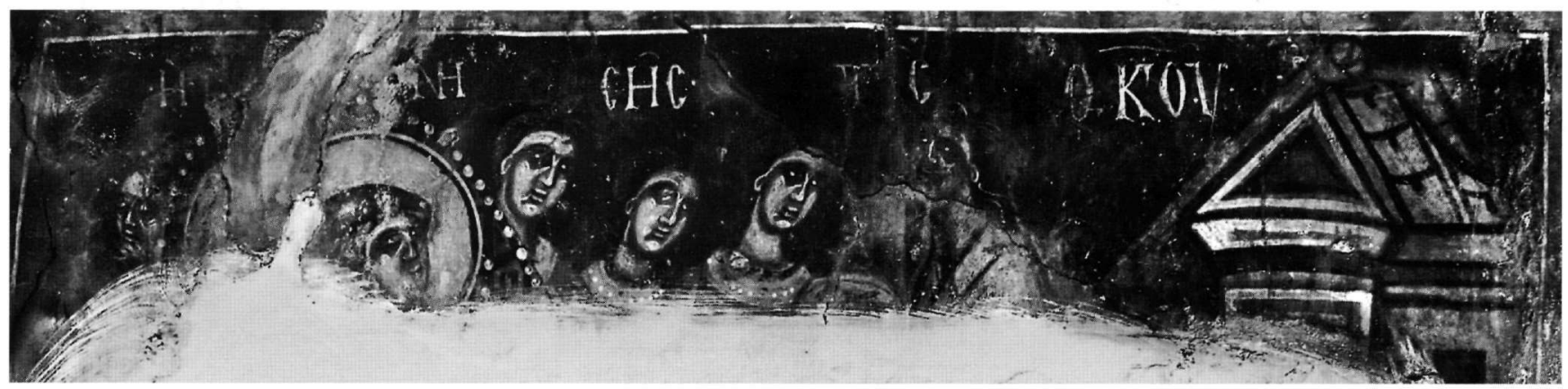

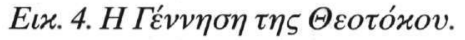

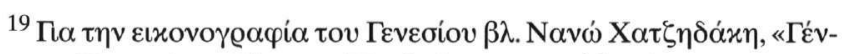

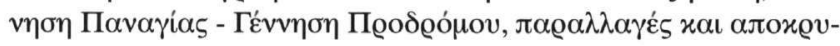

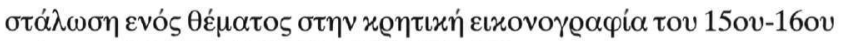

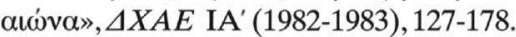




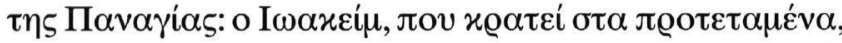

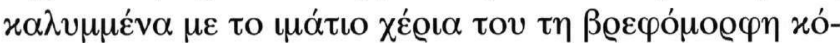

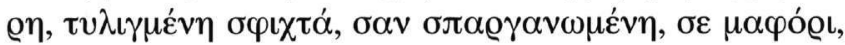

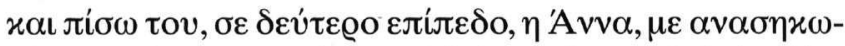

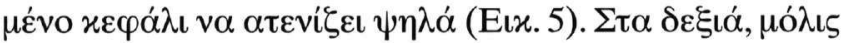

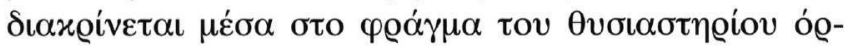

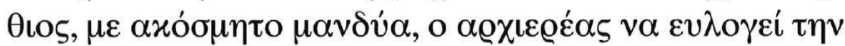

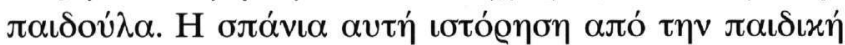

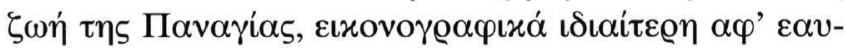

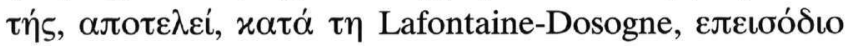

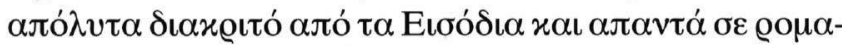

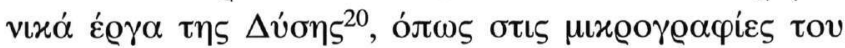

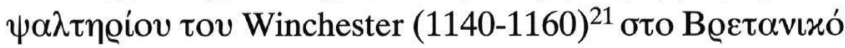

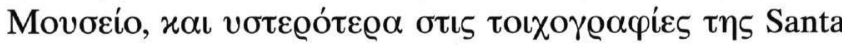

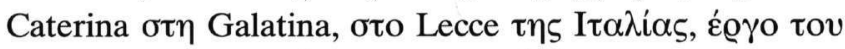

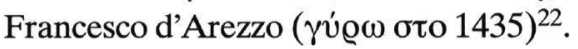

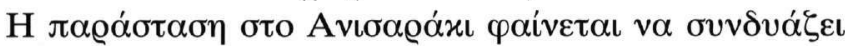

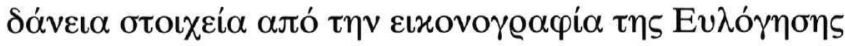

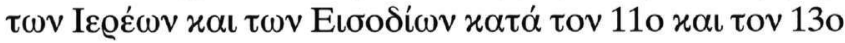

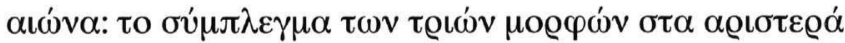

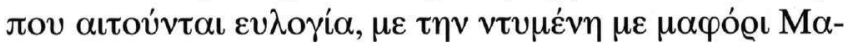

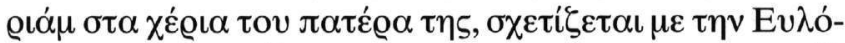

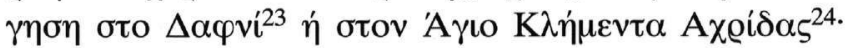

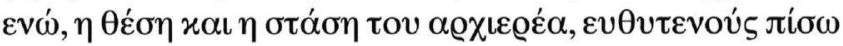

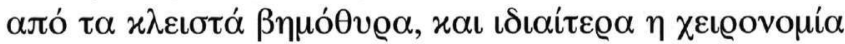

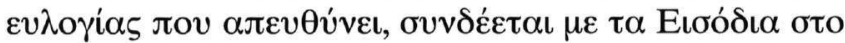

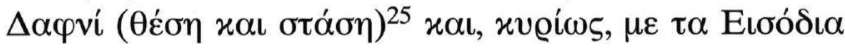

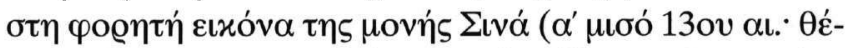

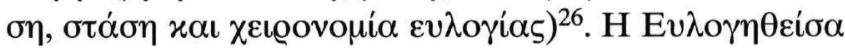

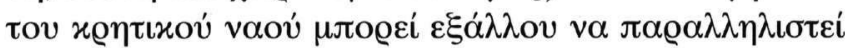

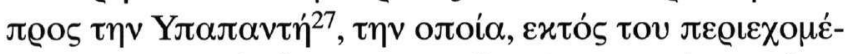

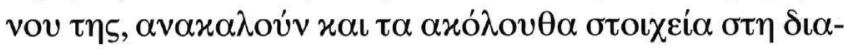

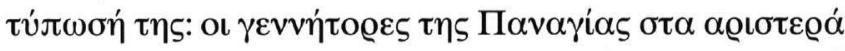

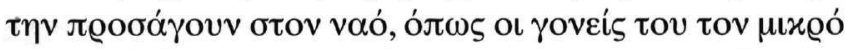

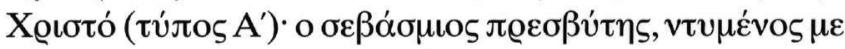

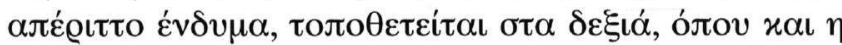

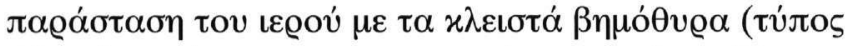

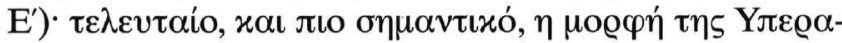

\footnotetext{
$20 \mathrm{~J}$. Lafontaine-Dosogne, Iconographie de l'enfance de la Vierge dans

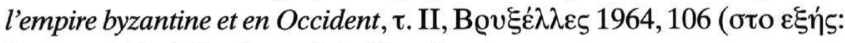
Iconographie de l'enfance de la Vierge).

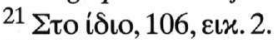

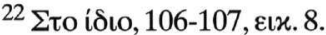

${ }^{23}$ Diez - Demus, Byzantine Mosaics, $\varepsilon\llcorner x .107$.

${ }^{24}$ Lafontaine-Dosogne, "Iconography”, $\varepsilon 1 x .16$.

${ }^{25}$ Diez - Demus, Byzantine Mosaics, $\varepsilon 1 x .110$.

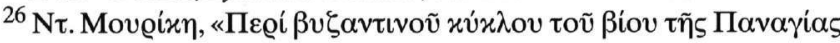

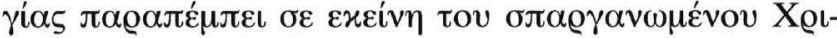

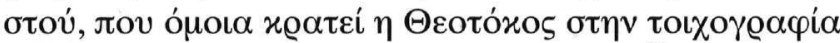

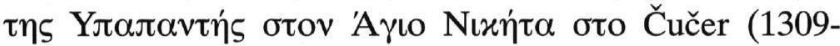

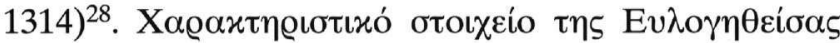

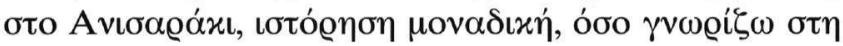

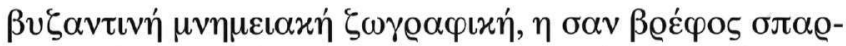

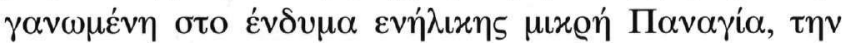

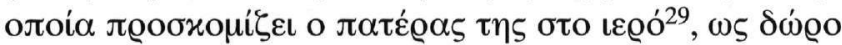

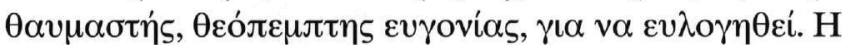

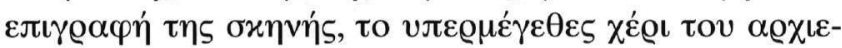

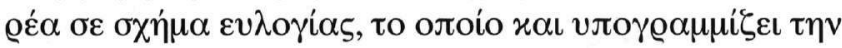

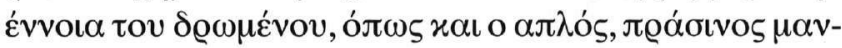

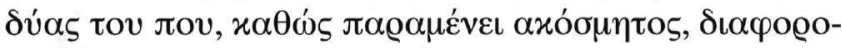

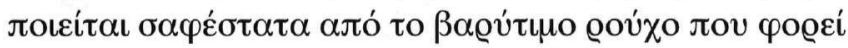

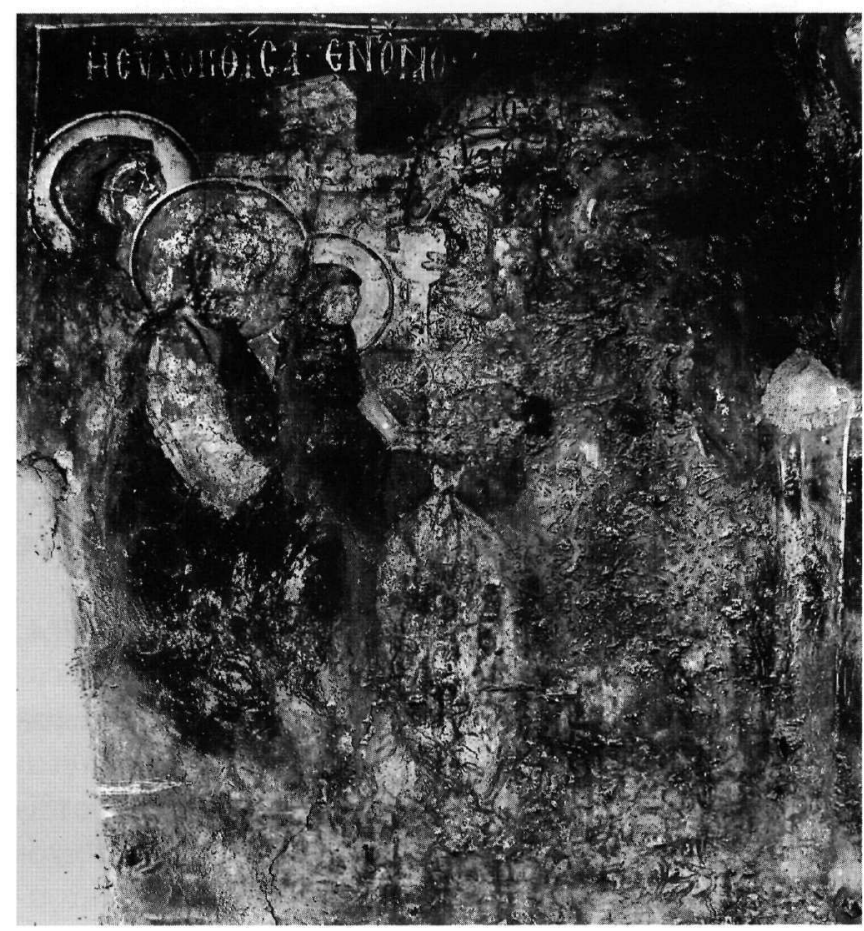

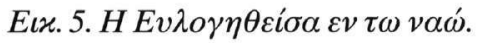

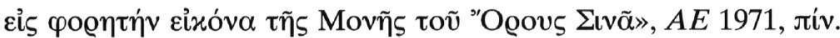

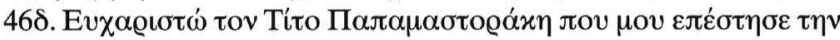

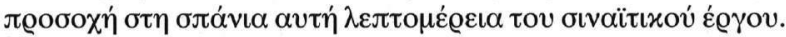

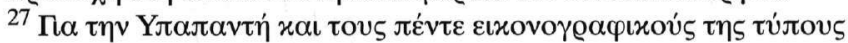

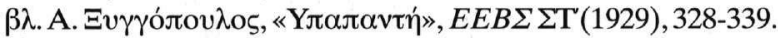

${ }^{28}$ Millet - Frolow, Yougoslavie, $\tau$. III, $\pi i$ iv. 36.4.

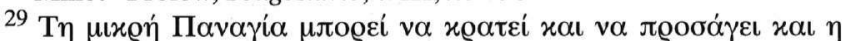

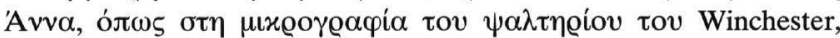
Lafontaine-Dosogne, Iconographie de l'enfance de la Vierge, عıx. 2. 


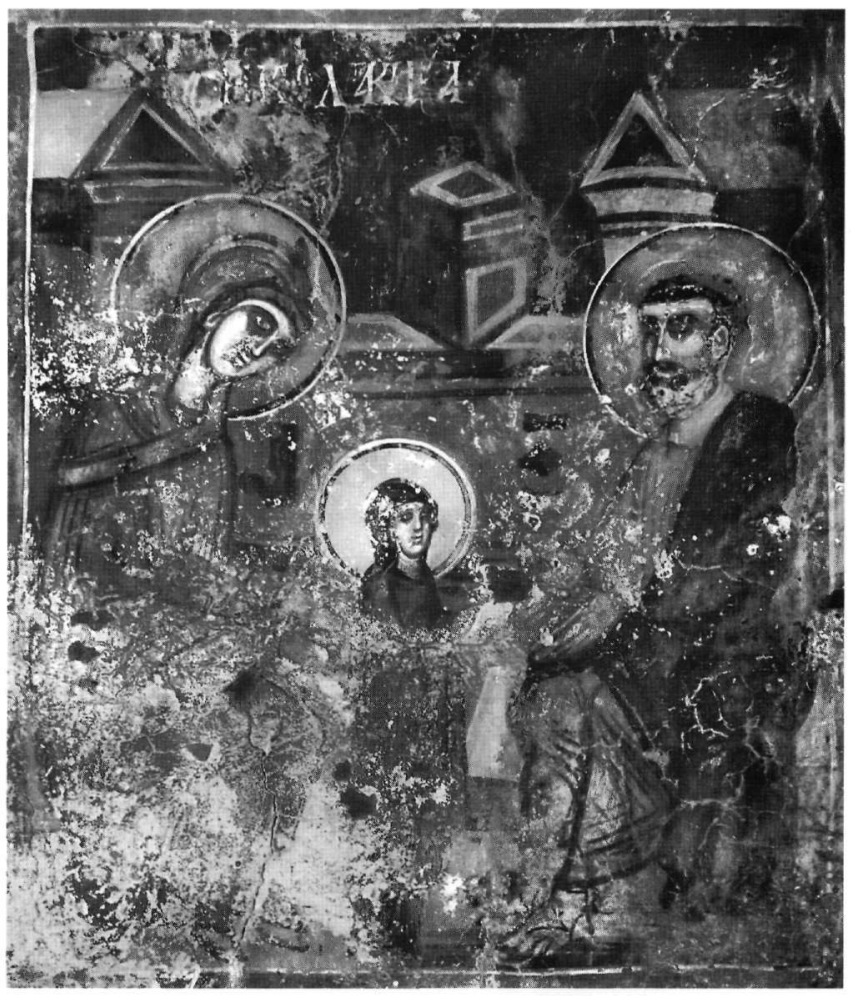

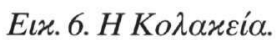

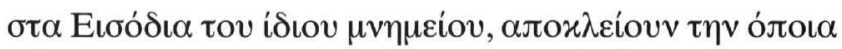

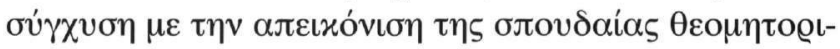

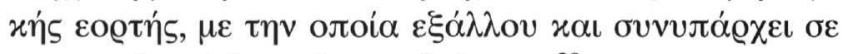

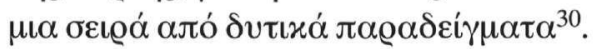

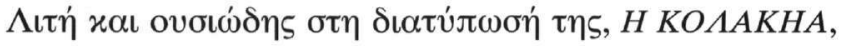

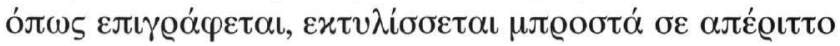

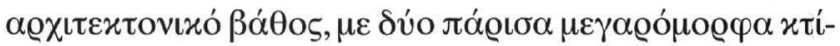

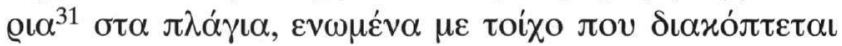

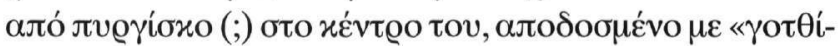

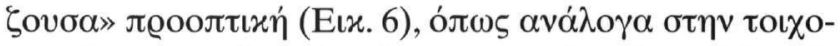

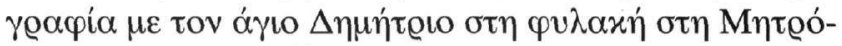

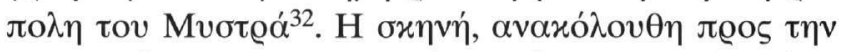

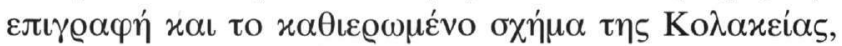

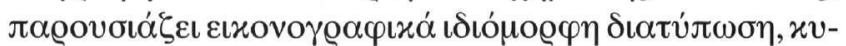

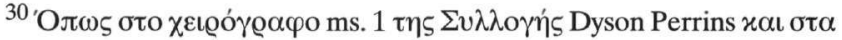

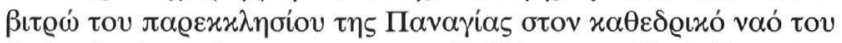

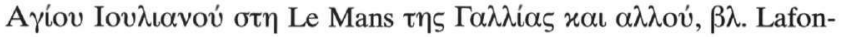
taine-Dosogne, Iconographie de l'enfance de la Vierge, 106.

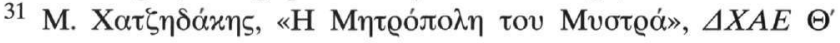

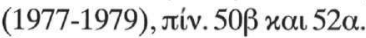

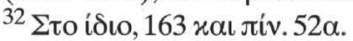

${ }^{33}$ Lafontaine-Dosogne, Iconographie de l'enfance de la Vierge, $\tau$. I, 123.
}

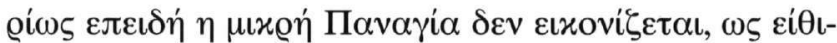

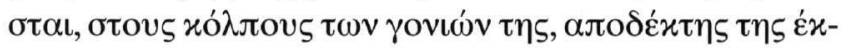

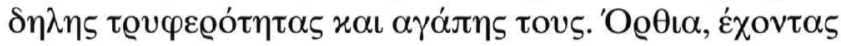

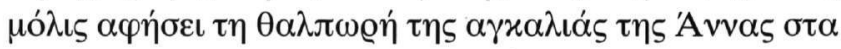

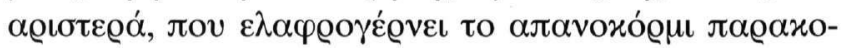

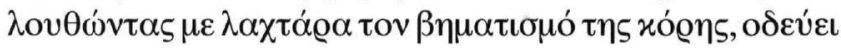

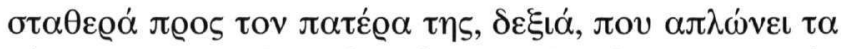

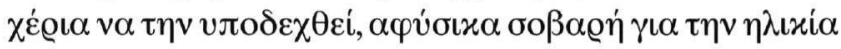

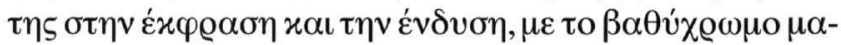

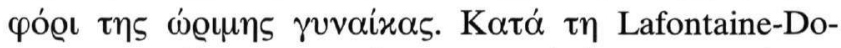

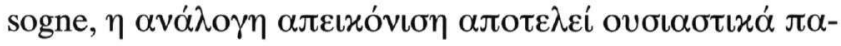

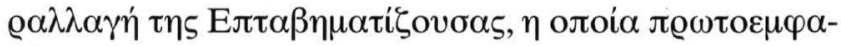

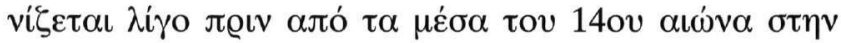

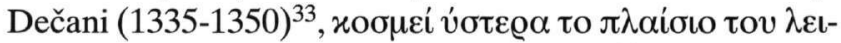

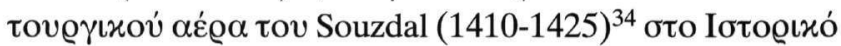

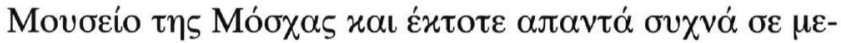

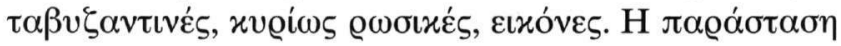

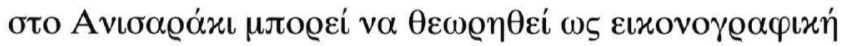

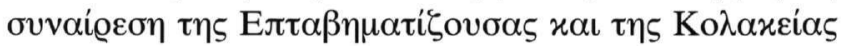

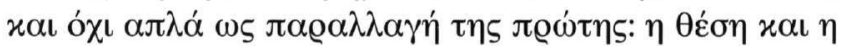

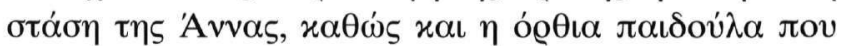

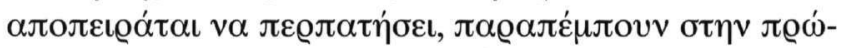

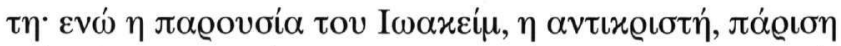

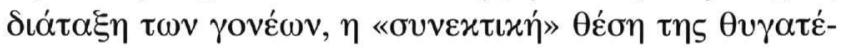

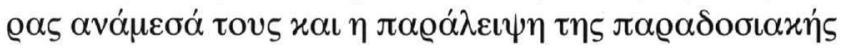

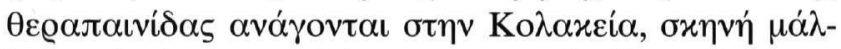

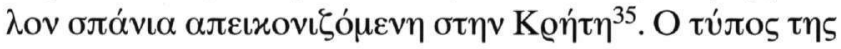

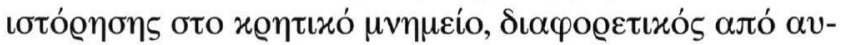

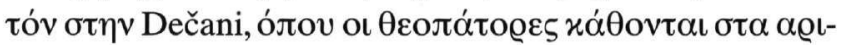

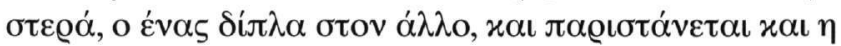

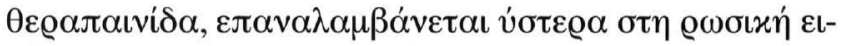

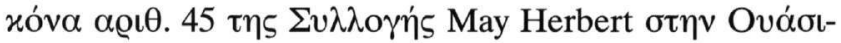

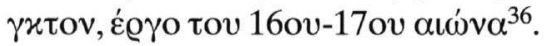

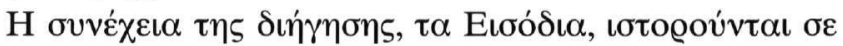

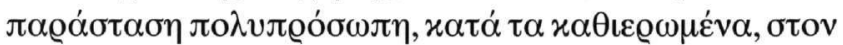

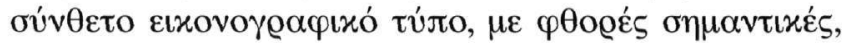

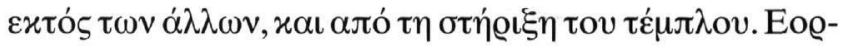

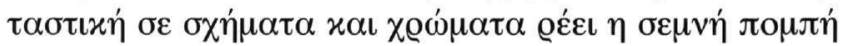

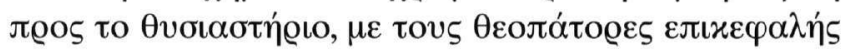

H íoı, «Les cycles de la Vierge dans l'eglise de Dečani: Enfance, Dormition et Akathiste», Dečani et l'art byzantin au milieu du XIVe

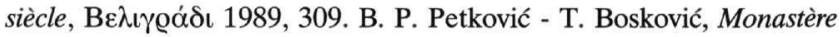

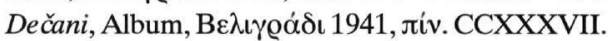

${ }^{34}$ Lafontaine-Dosogne, Iconographie de l'enfance de la Vierge, عıx. 24.

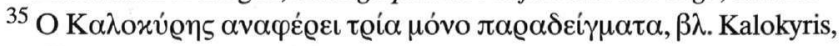
Wall Paintings of Crete, 122.

${ }^{36}$ Lafontaine-Dosogne, Iconographie de l'enfance de la Vierge, eıx. 33. 


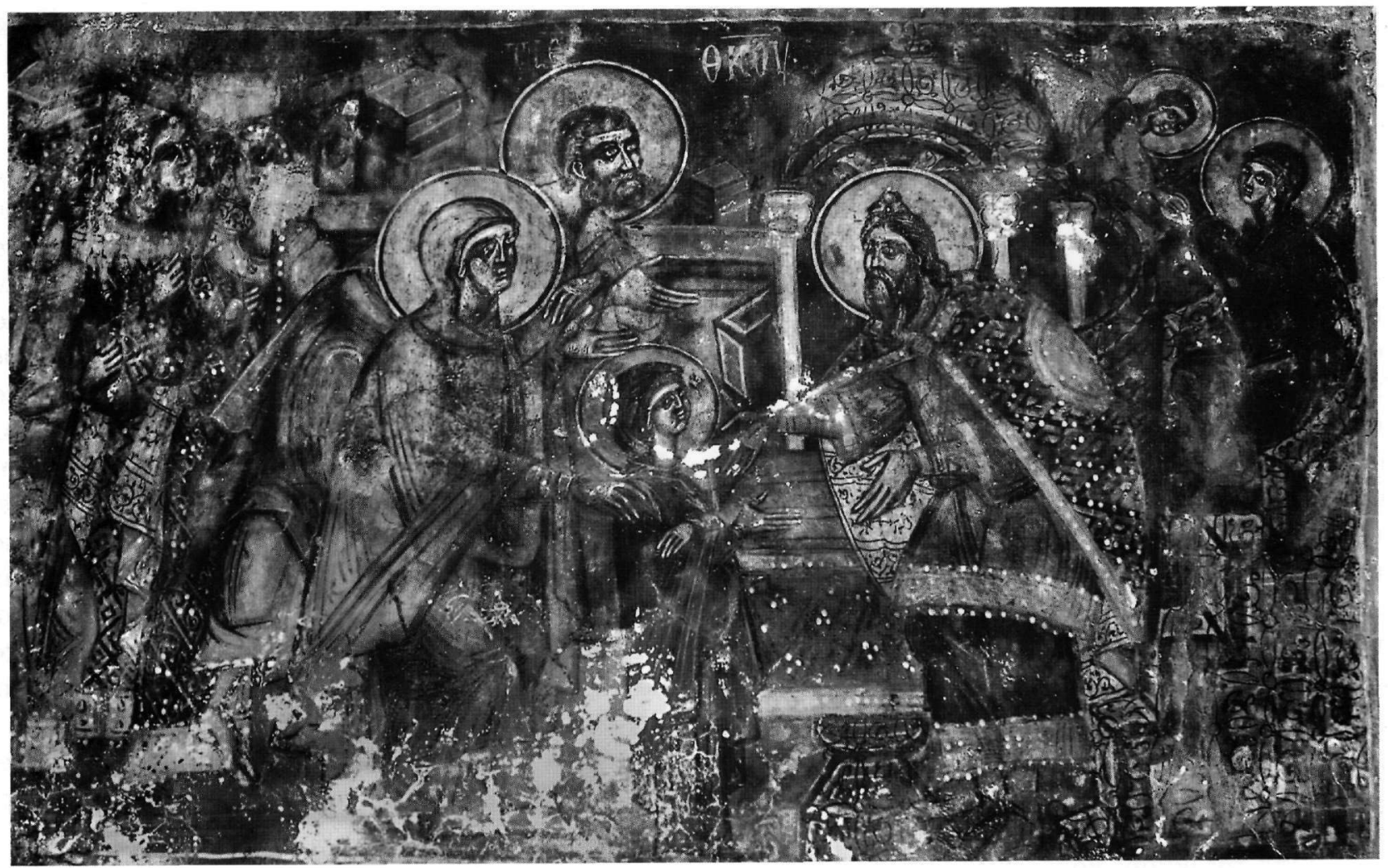

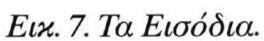

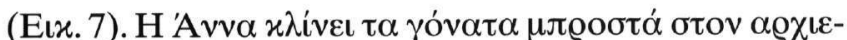

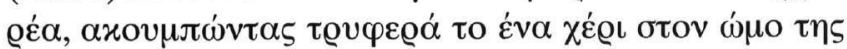

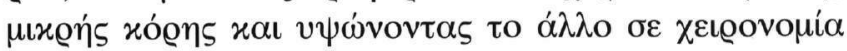

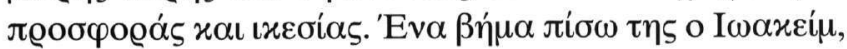

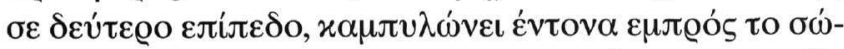

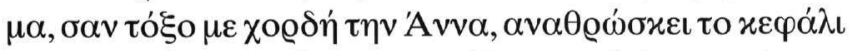

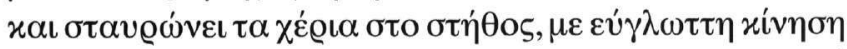

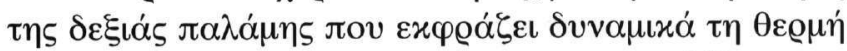

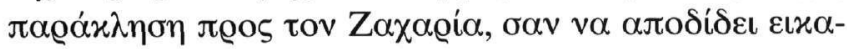

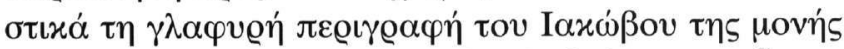

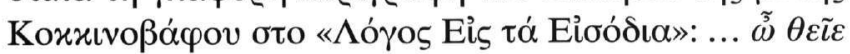

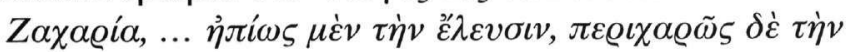

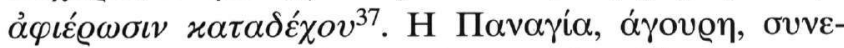

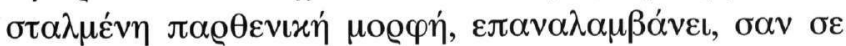

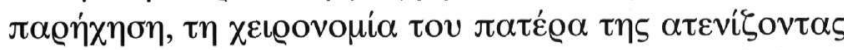

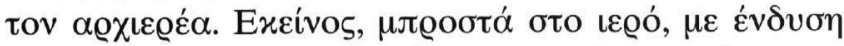

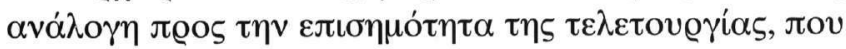

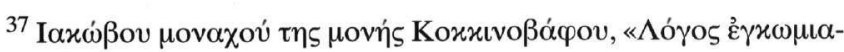

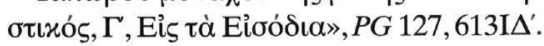

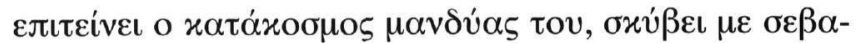

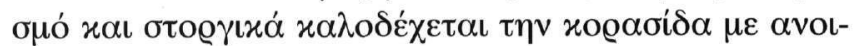

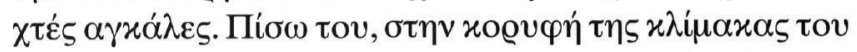

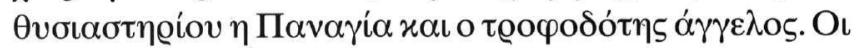

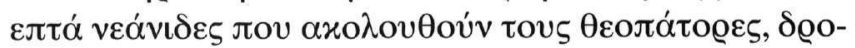

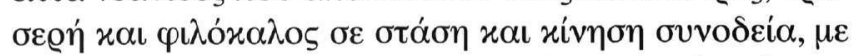

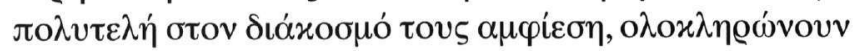

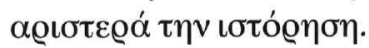

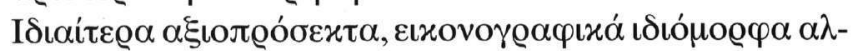

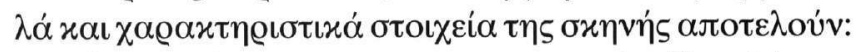

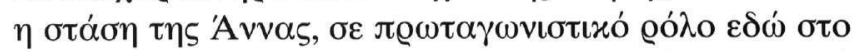

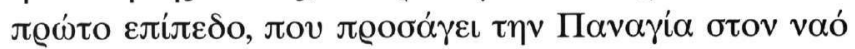

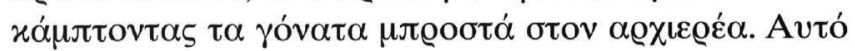

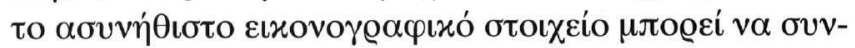

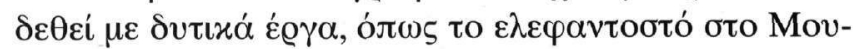

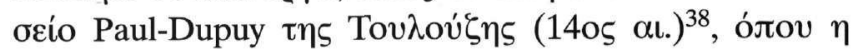

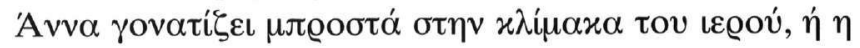

\footnotetext{
${ }^{38}$ Lafontaine-Dosogne, Iconographie de l'enfance de la Vierge, $\tau$. II, عเx. 51.
} 


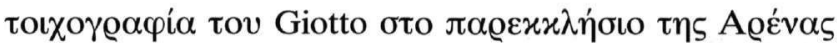

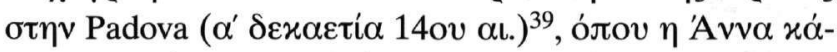

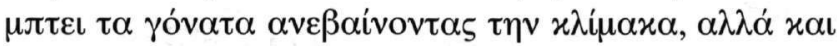

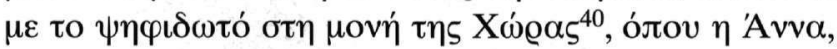

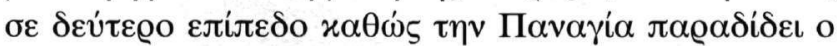

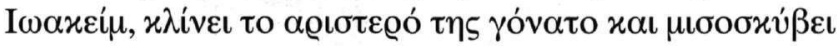

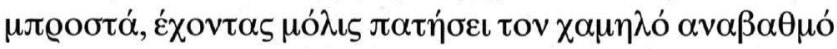

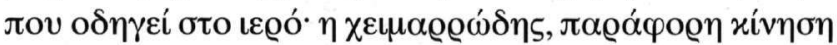

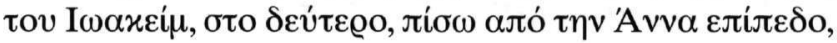

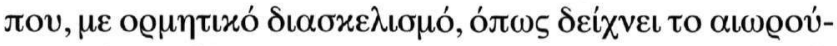

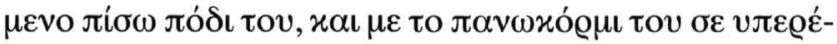

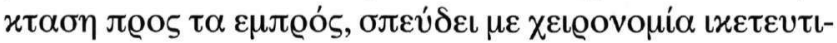

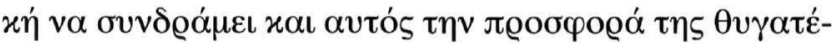

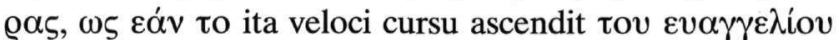

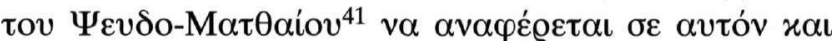

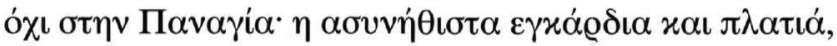

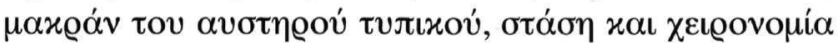

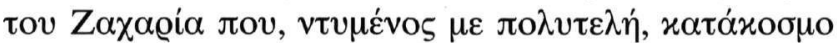

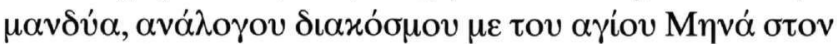

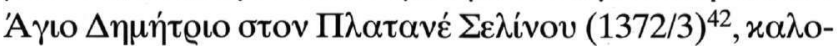

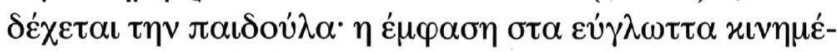

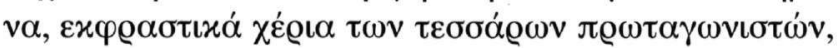

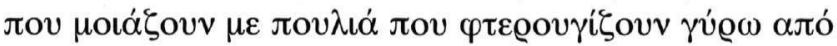

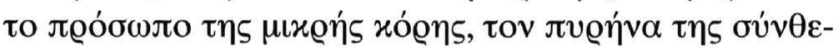

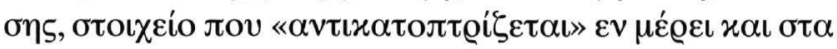

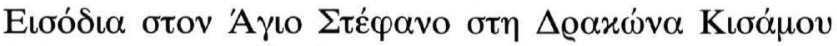

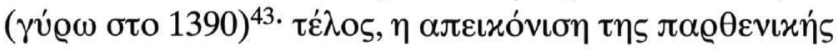

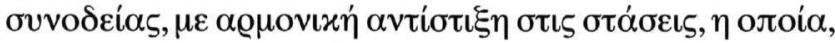

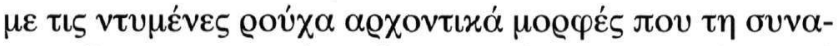

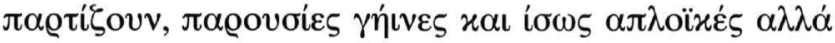

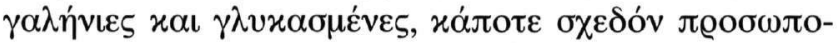

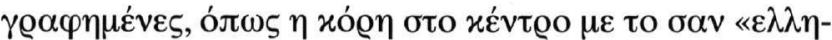

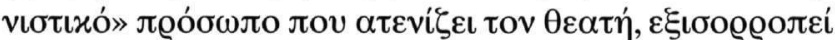

\footnotetext{
${ }^{39}$ M. Barasch, Giotto and the Language of Gesture, Cambridge, Mass. 1987, عเx. 43

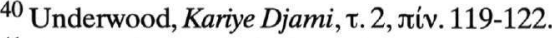

41 C. von Tischendorf, Evangelia Apocrypha, Pseudo-Matthaei Evange-

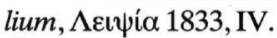

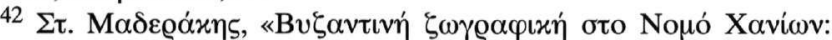

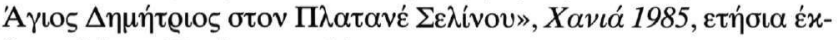

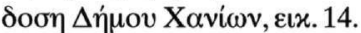

${ }^{43}$ Kalokyris, Wall Paintings of Crete, $\varepsilon\llcorner x$. BW88.

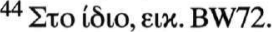

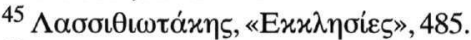

${ }^{46}$ M. Bissinger, Kreta. Byzantinische Wandmalerei, Móvaxo 1995, عıx. 57.

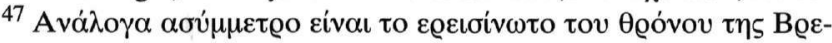

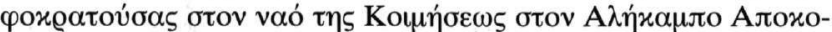

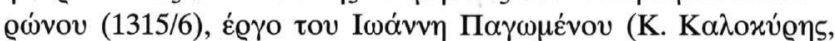

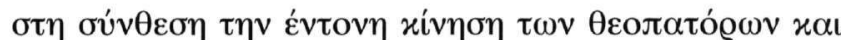

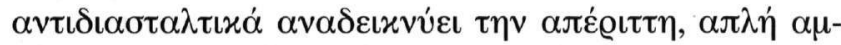

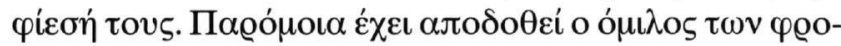

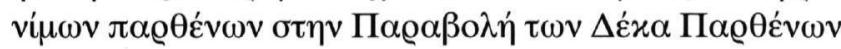

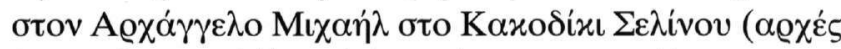

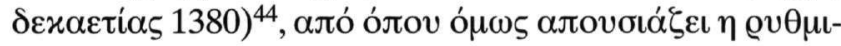

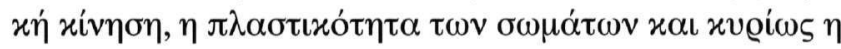

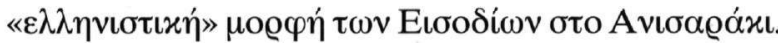

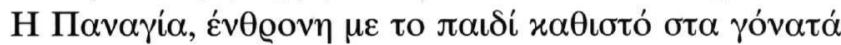

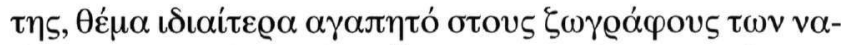

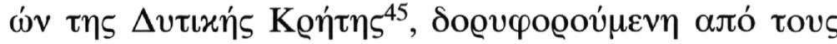

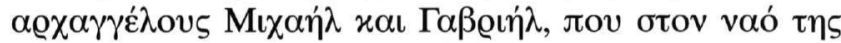

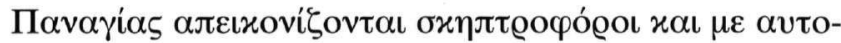

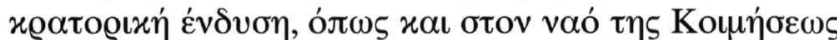

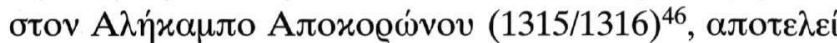

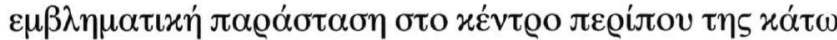

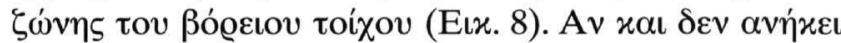

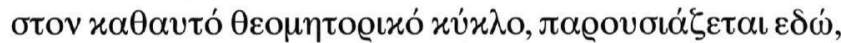

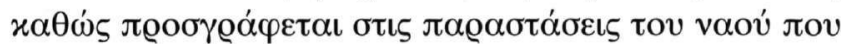

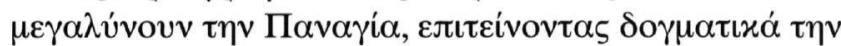

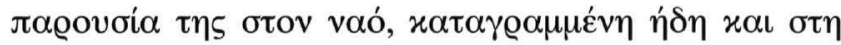

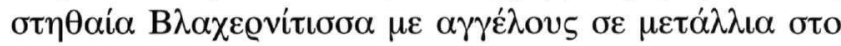

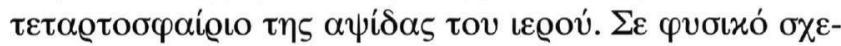

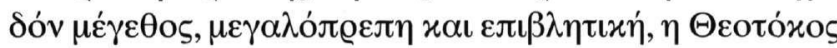

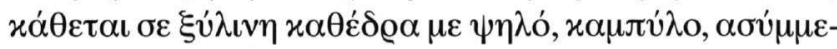

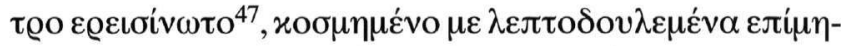

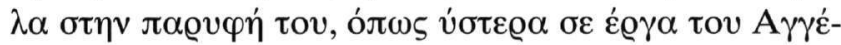

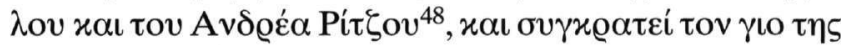

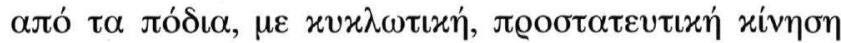

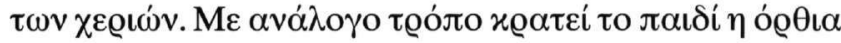

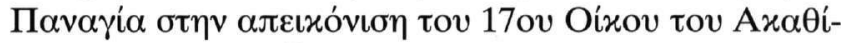

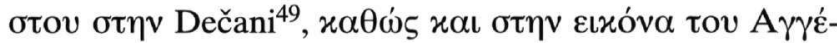

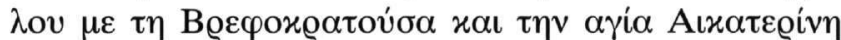




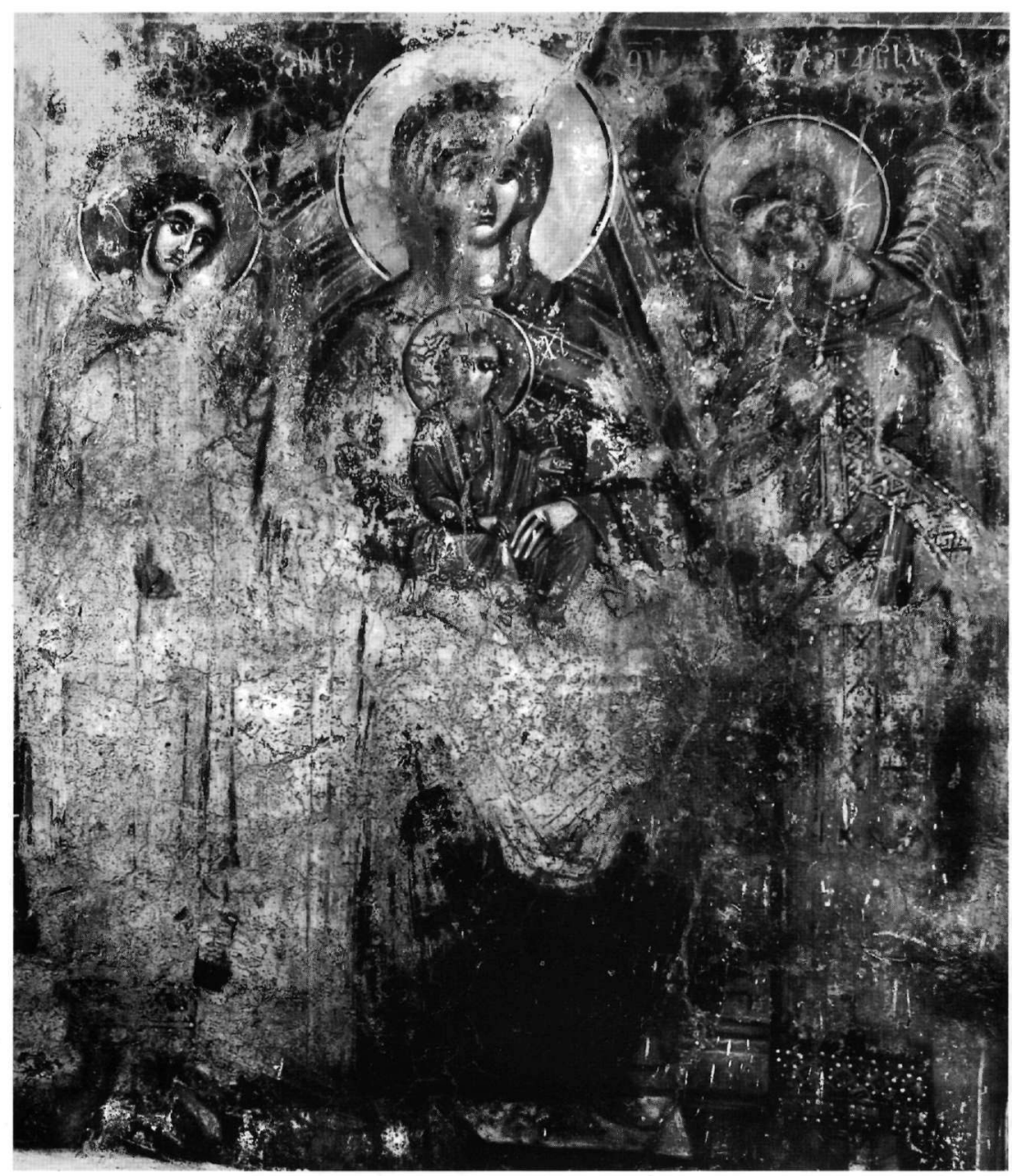

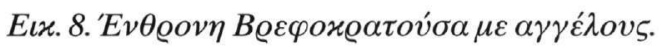

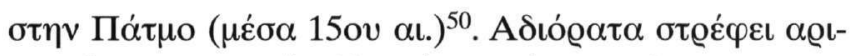

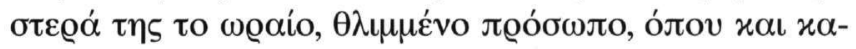

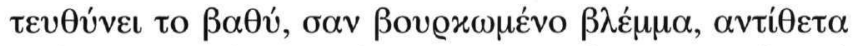

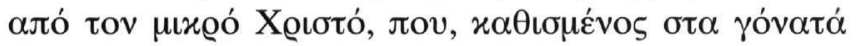

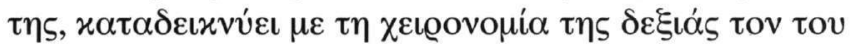

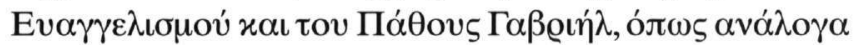

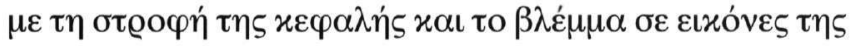

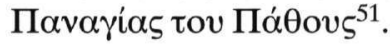

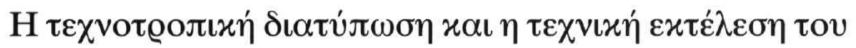

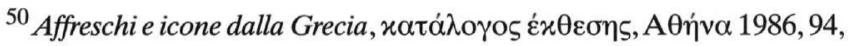
$\varepsilon เ x .54$ (M. Chatzidakis).

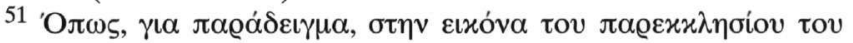

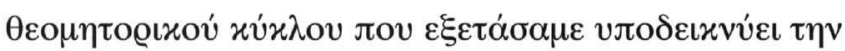

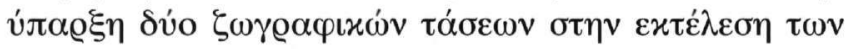

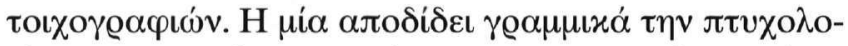

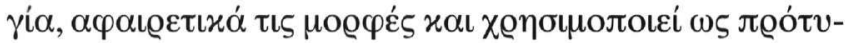

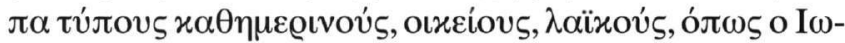

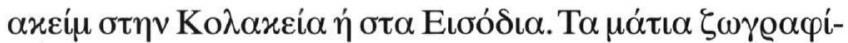

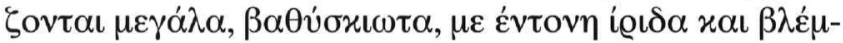

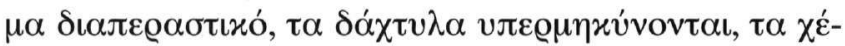

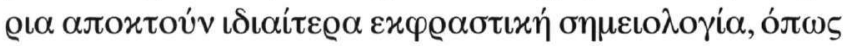

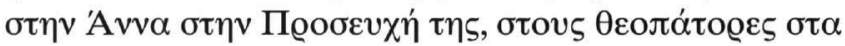

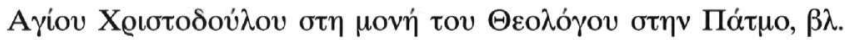

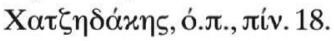




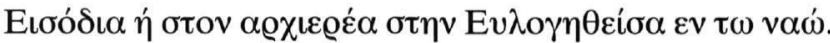

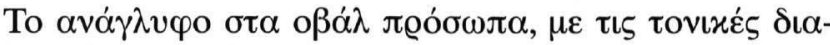

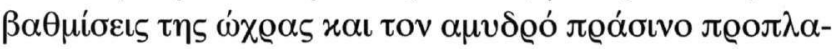

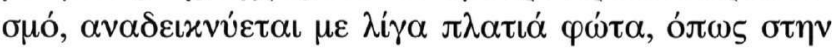
'A $v v \alpha \sigma \tau \eta v$ Ko $\lambda \alpha x \varepsilon i \alpha$.

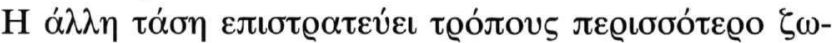

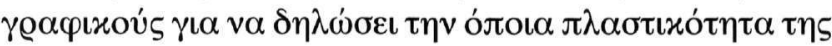

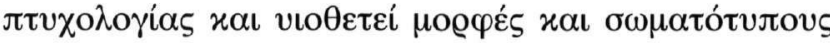

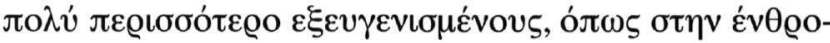

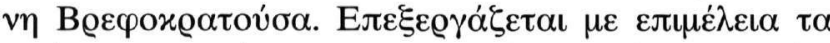

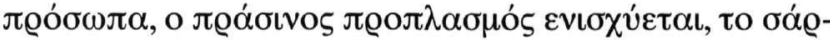

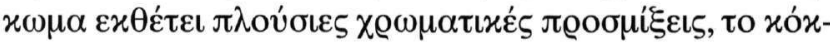

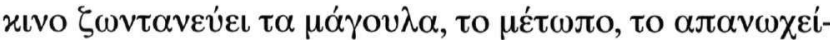

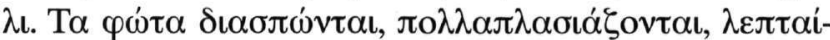

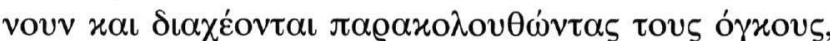

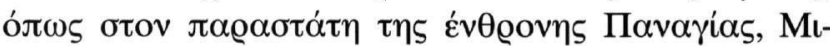

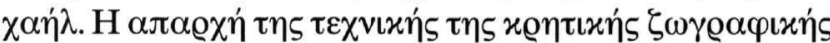

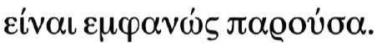

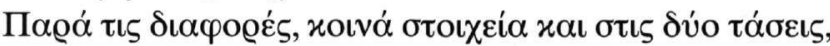

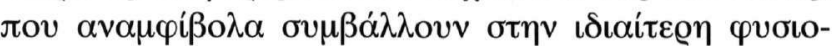

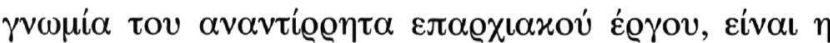

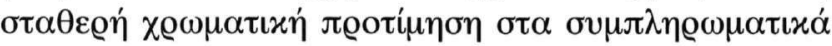

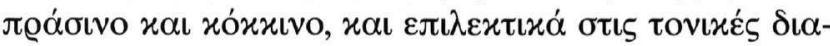

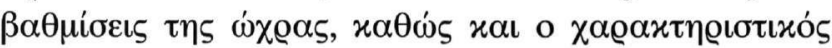

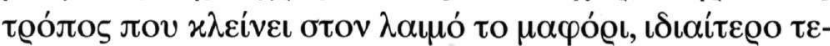

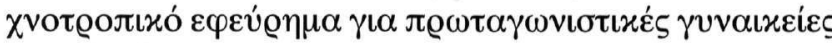

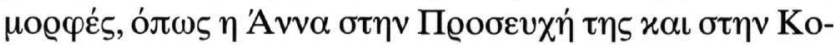

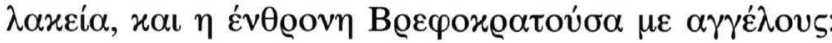

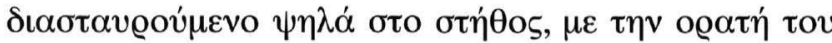

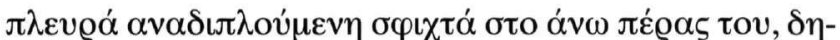

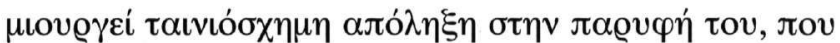

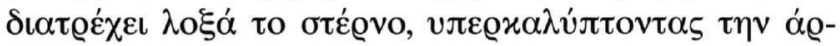

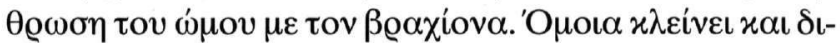

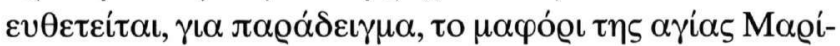

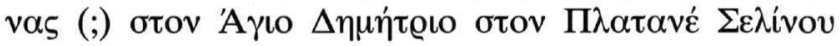

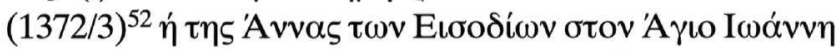

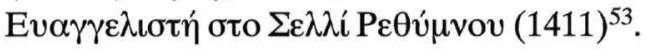

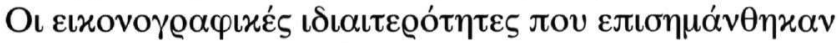

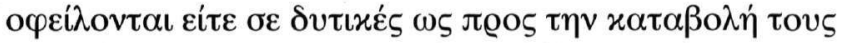

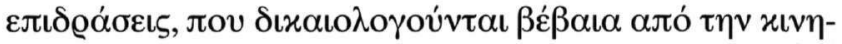

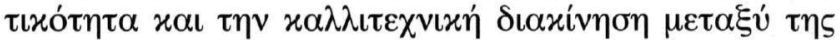

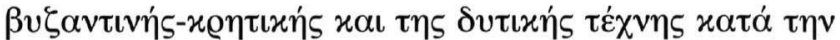

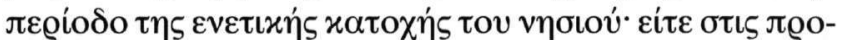

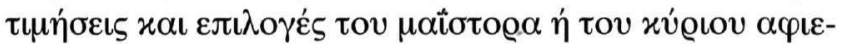

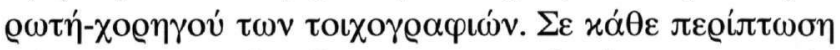

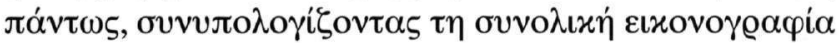

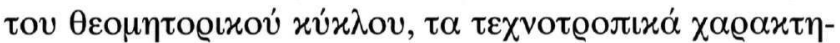

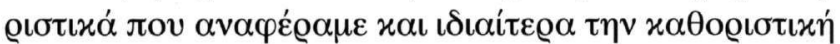

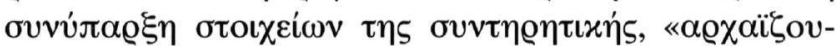

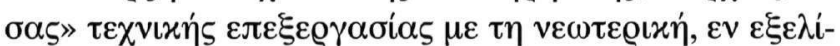

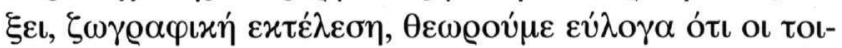

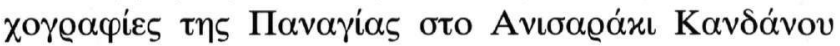

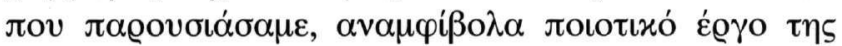

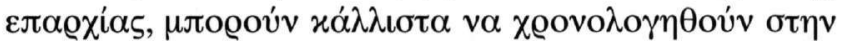

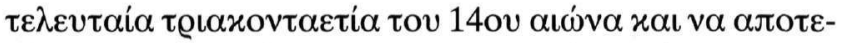

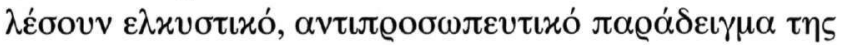

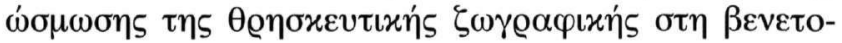

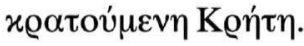

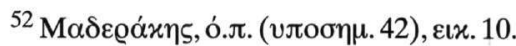

${ }^{53}$ Spatharakis, Dated Wall Paintings, عıx. 145. 


\section{Thetis Xanthaki}

\section{THE MARIOLOGICAL CYCLE IN THE CHURCH OF THE PANAYIA AT ANISARAKI, KANDANOS: UNUSUAL ICONOGRAPHIC FEATURES}

$\mathrm{T}$ he church of the Panayia at Anisaraki, Kandanos in the prefecture of Chania, with its uncluttered facade, is a small, aisleless church roofed with a pointed barrel vault. It belongs to the architectural type predominant in West Crete, from which it deviates only in the pointed dentilated bandcornice crowning its entrance on the exterior (Fig. 1) - a rare decorative element in the church-building of this prefecture. The wall-paintings of the lavishly decorated interior, though in a poor state of preservation, are highly interesting, with different elements coexisting and blending in their style and technique, and above all in their iconography.

The Mariological cycle in this church dedicated to the Virgin has the Virgin Blachernitissa with angels in bust as a frontispiece in the semidome of the sanctuary apse. It comprises a further five established episodes from her childhood - the Prayer of Joachim, the Prayer of Anne, the Birth of the Virgin and the Caresses of the Virgin, culminating in the impressively large Presentation of the Virgin in the Temple and ends with the almost entirely destroyed Dormition; It is enriched by the Virgin Blessed in the Temple, a scene that is very rare, if not without precedent, in Byzantine and PostByzantine monumental painting.

The very first, prefatory, scene of the Mariological cycle has an unusual feature, in that it depicts only the Lament-Prayer of Joachim (Fig. 2), without the established accompanying depiction of his Annunciation. The pose and expression of the grieving patriarch and of the bewildered, anxious shepherds looking on, the absence of the angel, and the name of the leading figure in the nominative $O I \Omega A K I M$ ('Joachim [in the Wilderness]'), which is all that survives of the inscription, all confirm that this is a representation of the Lamentation-Prayer.

The same feature also occurs in the H ПРОСЕYХH THC [AГIAC] ANHC ('Prayer of St Anne') (Fig. 3). The meaning of the scene is emphasised by the pose and expression of the saint and the maidservant accompanying her and, above all, by the very realistic, charming depiction of the nest with the four fledglings in the tree: with beaks wide open, they impa- tiently await the mother-nurse who hovers next to them, already feeding the first of them with the prey she holds in her beak.

The $H \Gamma[E] N H C H C T H C \Theta(E O T O) K O Y$ ('Birth of the Virgin') (Fig. 4) is badly damaged; in the part that survives, we may note the lavish decoration, with the almost realistic rendering of the pearls on Anne's partly preserved mattress; the large number of young women around the mother-to-be; and the general arrangement of the scene - all of which deviate from the model that was the basis for the formation of the Cretan iconography of the subject in the following, 15th, century.

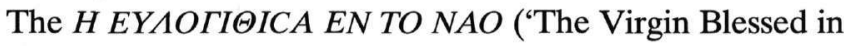
the Temple') (Fig. 5) is a rare scene from the Virgin's childhood which, according to Lafontaine-Dosogne, is completely distinct from the Presentation of the Virgin in the Temple, and is found in Romanesque and later works of the West, such as the wall-paintings of Santa Caterina at Lecce in Italy (c. 1435). The representation at Anisaraki combines loans from the Virgin Blessed by the Priests and the Presentation of the Virgin in the Temple (the former as formulated in St Clement in Ohrid, and the latter in a portable icon from Sinai dating from the first half of the 13 th century), and can be related in content, arrangement and basic iconographic features to the Presentation of Christ in the Temple. The inscription of the scene, the excessively large hand of the blessing priest, emphasising the meaning of the ritual, and his plain, undecorated vestment, preclude any confusion with the Presentation of the Virgin in the Temple, which is in any case found together with the present scene in the monument and also in a series of Western examples.

In the $H K O A A K H A$ ('Caresses of the Virgin') (Fig. 6), which does not follow the established scheme, the Virgin stands upright, having just left her mother's bosom, and is shown walking steadily towards Joachim, an iconographic feature that, according to Lafontaine-Dosogne, is a variation of the First Seven Steps and is first found at Dečani. The scene at Anisaraki may be regarded as a contraction of the 
First Seven Steps and the Caresses, rather than simply as a variation of the former: the position and stance of Anne and the little girl on her feet trying to walk recall the former; the presence of Joachim, the confronted figures of Joachim and Anne, the 'cohesive' position of their daughter between them, and the omission of the maidservant suggest the latter.

The main distinctive features of the Presentation of the Virgin in the Temple (Fig. 7) may be summed up in the stance of Anne who, bringing her daughter to the temple, kneels before the high priest - an unusual iconographic element to be found in Western works, such as the Toulouse ivory (14th century) or the chapel of the Arena at Padua (first decade of the 14th century), and also in Byzantine art, as in the mosaic in the Chora Monastery; in the impulsive movement of $\mathrm{Za}$ charias; in the eloquent expressive gestures of the arms of the four protagonists, who resemble a flock of birds around the Virgin's face; and finally, in the splendid, long retinue of maidens with their luxurious dress and rhythmical movement, which is highlighted by the emblematic figure (almost a portrait) of the girl with the 'Hellenistic' face at the centre. The majestic depiction of the enthroned Virgin and Child with angels (Fig. 8), which gives greater doctrinal intensity to the Virgin's presence in the church, has compositional features that are to be found at Dečani, in 14th-century Cretan monuments, and later in works by Angelos and Andreas Ritzos, as well as in icons of the Virgin of the Passion. These features include the way in which the mother holds the child, the shape, rendering and decoration of the throne, and the complex relationship between the figure of the Christ Child and Gabriel, the archangel of the Passion.

The unusual iconographic elements and the characteristic preferences noted above are due either to influences of Western origin, which can be accounted for, of course, either by movement and trade between Byzantine-Cretan and Western art during the period of Venetian rule on the island; or by preferences and choices of the master painter or the donor-sponsor of the wall-paintings. In any case, taking into account the overall iconography of the Mariological cycle and attendant motifs, the style of the wall-paintings, and particularly the definitive coexistence of features of the conservative, 'archaising' technique alongside the newer, developing, style of painting, it seems reasonable to suggest that the wall-paintings of the Virgin at Anisaraki, Kandanos, undoubtedly a qualitative work of the periphery, should be dated to the last thirty years of the 14th century, forming an attractive, representative example of the osmosis of religious painting in Crete under the Venetians. 